T. OTSUKI

KODAI MATH. J.

4 (1981), 28-70

\title{
A CERTAIN PROPERTY OF GEODESICS OF THE FAMILY OF RIEMANNIAN MANIFOLDS $O_{n}^{2}$ (III)
}

\author{
By Tominosuke OTsuki
}

\section{§. Introduction.}

This is a continuation of Part (I) and Part (II) with the same title written by the present author. We shall use the same notation in them.

The period $T$ of any non-constant solution $x(t)$ of the non-linear differential equation of order 2 :

$$
n x\left(1-x^{2}\right) \frac{d^{2} x}{d t^{2}}+\left(\frac{d x}{d t}\right)^{2}+\left(1-x^{2}\right)\left(n x^{2}-1\right)=0
$$

with a constant $n>1$ such that $x^{2}+x^{\prime 2}<1$ is given by the integral:

$$
T=\sqrt{n c} \int_{x_{0}}^{x_{1}} \frac{d x}{x \sqrt{(n-x)\left\{x(n-x)^{n-1}-c\right\}}},
$$

where $0<x_{0}<1<x_{1}<n$ and $c=x_{0}\left(n-x_{0}\right)^{n-1}=x_{1}\left(n-x_{1}\right)^{n-1}$.

We shall study the following conjecture in the present work, which $1 \mathrm{~s}$ in place of Conjecture B in Part (II), implying the inequality:

$$
T<\sqrt{2} \pi .
$$

Conjecture C. The period function $T$ as a function of $\tau=\left(x_{1}-1\right) /(n-1)$ and $n$ is monotone decreasing with respect to $n(\geqq 2)$ for any fixed $\tau(0<\tau<1)$.

\section{$\S 1$. Fundamental formulas.}

Using a closed curve $\gamma$ on the Riemann surface $\mathscr{I}: z(n-z)^{n-1}-w^{2}=c$ as in [11], $T$ can be written as

$$
T=T(c, n)=-\frac{\sqrt{n c}}{2} \int_{r} \frac{d z}{z \sqrt{(n-z)}\left\{z(n-z)^{n-1}-c\right\}} .
$$

from which we obtain

$$
\frac{\partial T}{\partial c}=-\frac{1}{4} \sqrt{\frac{n}{c}} \int_{r} \frac{(n-z)^{n-3 / 2} d z}{\sqrt{\left\{z(n-z)^{n-1}-c\right\}^{3}}}
$$

and 


$$
\begin{aligned}
\frac{\partial T}{\partial n}= & -\frac{1}{4} \sqrt{\frac{c}{n}} \int_{r} \frac{d z}{z \sqrt{(n-z)\left\{z(n-z)^{n-1}-c\right\}}} \\
& +\frac{\sqrt{n c}}{4} \int_{r} \frac{1}{z \sqrt{(n-z)\left\{z(n-z)^{n-1}-c\right\}}}\left[\frac{1}{n-z}+\frac{z(n-z)^{n-1} \lambda(z)}{z(n-z)^{n-1}-c}\right] d z,
\end{aligned}
$$

where

$$
\lambda(z)=\log (n-z)+\frac{n-1}{n-z} .
$$

(See $\S 1$ in Part (I)).

Now, we denote $T$ by $\Omega(\tau, n)$, considering it as a function of $\tau$ and $n$. From $\tau=\left(n-x_{1}\right) /(n-1)$, we obtain

$$
x_{1}=\tau+n-\tau n
$$

From $c=x_{1}\left(n-x_{1}\right)^{n-1}$, we obtain

$$
\frac{\partial c}{\partial \tau}=\frac{\partial c}{\partial x_{1}} \frac{\partial x_{1}}{\partial \tau}=n(n-1)\left(x_{1}-1\right)\left(n-x_{1}\right)^{n-2}
$$

and

$$
\begin{aligned}
\frac{\partial c}{\partial n} & =\frac{\partial c}{\partial x_{1}} \frac{\partial x_{1}}{\partial n}+\frac{\partial c}{\partial n} \\
& =n\left(1-x_{1}\right)\left(n-x_{1}\right)^{n-2}(1-\tau)+c \lambda\left(x_{1}\right) \\
& =-\frac{n\left(x_{1}-1\right)^{2}\left(n-x_{1}\right)^{n-2}}{n-1}+c \lambda\left(x_{1}\right) .
\end{aligned}
$$

By (1.2) and (1.3), we obtain

$$
\begin{aligned}
& \frac{\partial \Omega(\tau, n)}{\partial n}=\frac{\partial T(c, n)}{\partial c} \frac{\partial c}{\partial n}+\frac{\partial T(c, n)}{\partial n} \\
= & -\frac{1}{4} \sqrt{\frac{n}{c}}\left\{-\frac{n\left(x_{1}-1\right)^{2}\left(n-x_{1}\right)^{n-2}}{n-1}+c \lambda\left(x_{1}\right)\right\} \int_{r} \frac{(n-z)^{n-3 / 2} d z}{\sqrt{\{\phi(z)-c\}^{3}}} \\
& -\frac{1}{4} \sqrt{\frac{c}{n}} \int_{r} \frac{d z}{z \sqrt{(n-z)\{\phi(z)-c\}}} \\
& +\frac{\sqrt{n c}}{4} \int_{r} \frac{1}{z \sqrt{(n-z)\{\phi(z)-c\}}}\left[\frac{1}{n-z}+\frac{\phi(z) \lambda(z)}{\phi(z)-c}\right] d z,
\end{aligned}
$$

where

$$
\phi(z)=z(n-z)^{n-1} .
$$

Furthermore, this can be written as

$$
\frac{\partial \Omega(\tau, n)}{\partial n}=\frac{n \sqrt{n}}{4 \sqrt{ } c}=\frac{\left(x_{1}-1\right)^{2}\left(n-x_{1}\right)^{n-2}}{n-1} \int_{r} \frac{(n-z)^{n-3 / 2} d z}{\sqrt{\{\phi(z)-c\}^{3}}}
$$




$$
+\frac{\sqrt{n c}}{4} \int_{r} \frac{(n-z)^{n-3 / 2}\left\{\lambda(z)-\lambda\left(x_{1}\right)\right\} d z}{\sqrt{\{\phi(z)-c\}^{3}}}+\frac{\sqrt{c}}{4 \sqrt{n}} \int_{r} \frac{d z}{\sqrt{(n-z)^{3}\{\phi(z)-c\}}}
$$

The 1st term in the right hand side is negative by Proposition 1 in [10] or Theorem D in [12] and the 3rd term is also negative because

$$
\int_{\gamma} \frac{d z}{\sqrt{(n-z)^{3}\{\phi(z)-c\}}}=-2 \int_{x_{0}}^{x_{1}} \frac{d x}{\sqrt{(n-x)^{3}\left\{x(n-x)^{n-1}-c\right\}}}<0 .
$$

In the following, we shall change the right hand side of (1.6) into a real integral.

Setting

$$
\begin{aligned}
& J_{1}(\gamma):=\int_{\gamma} \frac{(n-z)^{n-3 / 2}\left\{\lambda(z)-\lambda\left(x_{1}\right)\right\} d z}{\sqrt{\left\{z(n-z)^{n-1}-c\right\}^{3}}}, \\
& J_{2}(\gamma):=\int_{\gamma} \frac{d z}{\sqrt{(n-z)^{3}\left\{z(n-z)^{n-1}-c\right\}}}, \\
& J_{3}(\gamma):=\int_{\gamma} \frac{(n-z)^{n-3 / 2} d z}{\sqrt{\left\{z(n-z)^{n-1}-c\right\}^{3}}},
\end{aligned}
$$

(1.6) can be written as

$$
\frac{\partial \Omega(\tau, n)}{\partial n}=\frac{\sqrt{c}}{4 \sqrt{n}}\left[\left\{n J_{1}(\gamma)+J_{2}(\gamma)\right\}+\frac{n^{2}\left(x_{1}-1\right)^{2}\left(n-x_{1}\right)^{n-2}}{(n-1) c} J_{3}(\gamma)\right] .
$$

Since the Riemann surface $\mathscr{F}$ is defined by the equation:

$$
z(n-z)^{n-1}-w^{2}=c,
$$

we have on $\mathscr{I}$ the equality:

and so

$$
n(1-z)(n-z)^{n-2} d z=2 w d w
$$

$$
\begin{aligned}
& \frac{(n-z)^{n-3 / 2}\left\{\lambda(z)-\lambda\left(x_{1}\right)\right\} d z}{\sqrt{\left\{z(n-z)^{n-1}-c\right\}^{3}}}=\frac{2}{n} \frac{\sqrt{n-z}\left\{\lambda(z)-\lambda\left(x_{1}\right)\right\}}{1-z} \cdot \frac{d w}{w^{2}} \\
= & -\frac{2}{n} d\left[\frac{\sqrt{n-z}\left\{\lambda(z)-\lambda\left(x_{1}\right)\right\}}{1-z} \cdot \frac{1}{w}\right] \\
& +\frac{2}{n} \frac{1}{w}\left[-\frac{1}{\sqrt{(n-z)^{3}}}+\left\{\lambda(z)-\lambda\left(x_{1}\right)\right\}\left\{\frac{\sqrt{n-z}}{(1-z)^{2}}-\frac{1}{2(1-z) \sqrt{n-z}}\right\}\right] d z \\
= & -\frac{2}{n} d\left[\frac{\sqrt{n-z}\left\{\lambda(z)-\lambda\left(x_{1}\right)\right\}}{1-z} \cdot \frac{1}{w}\right]-\frac{2}{n} \frac{d z}{\sqrt{(n-z)^{3}}\left\{z(n-z)^{n-1}-c\right\}} \\
& +\frac{(2 n-1-z)\left\{\lambda(z)-\lambda\left(x_{1}\right)\right\} d z}{n(1-z)^{2} \sqrt{(n-z)\left\{z(n-z)^{n-1}-c\right\}}} .
\end{aligned}
$$

Now, we divide $\gamma$ into the subarcs $\gamma^{\prime}$ and $\gamma^{\prime \prime}$ by taking a real constant $a$ $\left(x_{0}<a<1\right)$ as is shown in Fig. 1. 


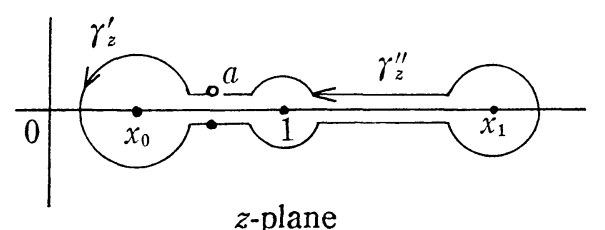

$z$-plane

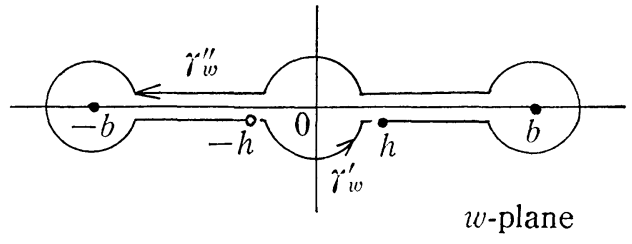

$\left(b=\sqrt{(n-1)^{n-1}-c}, h=\sqrt{a(n-a)^{n-1}-c}\right)$

Fig. 1.

Then, we have

$$
\begin{aligned}
& J_{1}(\gamma)=J_{1}\left(\gamma^{\prime}\right)+J_{1}\left(\gamma^{\prime \prime}\right), \\
& J_{1}\left(\gamma^{\prime}\right)=-\frac{2}{n}\left[\frac{\sqrt{n-z}\left\{\lambda(z)-\lambda\left(x_{1}\right)\right\}}{1-z} \cdot \frac{1}{w}\right]_{\partial \gamma^{\prime}} \\
& -\frac{2}{n} \int_{r^{\prime}} \frac{d z}{\sqrt{(n-z)^{3}}\left\{z(n-z)^{n-1}-c\right\}} \\
& +\frac{1}{n} \int_{r^{\prime}} \frac{(2 n-1-z)\left\{\lambda(z)-\lambda\left(x_{1}\right)\right\} d z}{(1-z)^{2} \sqrt{(n-z)}\left\{\overline{z(n-z)^{n-1}-c}\right\}} \\
& =-\frac{4}{n} \frac{\sqrt{n-a}\left\{\lambda\left(x_{1}\right)-\lambda(a)\right\}}{(1-a) \sqrt{a(n-a)^{n-1}-c}}+\frac{4}{n} \int_{x_{0}}^{a} \frac{d x}{\sqrt{(n-x)^{3}\left\{x(n-x)^{n-1}-c\right\}}} \\
& +\frac{2}{n} \int_{x_{0}}^{a} \frac{(2 n-1-x)\left\{\lambda\left(x_{1}\right)-\lambda(x)\right\} d x}{(1-x)^{2} \sqrt{(n-x)\left\{x(n-x)^{n-1}-c\right\}}}
\end{aligned}
$$

and

$$
J_{1}\left(\gamma^{\prime \prime}\right)=2 \int_{a}^{x_{1}} \frac{(n-x)^{n}\left\{\lambda\left(x_{1}\right)-\lambda(x)\right\} d x}{\sqrt{(n-x)^{3}\left\{x(n-x)^{n-1}-c\right\}^{3}}} .
$$

Hence, from these equalities and (1.7), we obtain

$$
\begin{aligned}
n J_{1}(\gamma)+J_{2}(\gamma)= & -\frac{4 \sqrt{n-a}\left\{\lambda\left(x_{1}\right)-\lambda(a)\right\}}{(1-a) \sqrt{a(n-a)^{n-1}-c}} \\
& +2 \int_{x_{0}}^{a} \frac{(1-x)^{2}+(n-x)(2 n-1-x)\left\{\lambda\left(x_{1}\right)-\lambda(x)\right\}}{(1-x)^{2} \sqrt{(n-x)^{3}}\left\{x(n-x)^{n-1}-c\right\}} d x \\
& +2 \int_{a}^{x_{1}} \frac{-x(n-x)^{n-1}+c+n(n-x)^{n}\left\{\lambda\left(x_{1}\right)-\lambda(x)\right\}}{\sqrt{(n-x)^{3}\left\{x(n-x)^{n-1}-c\right\}^{3}}} .
\end{aligned}
$$

The 2 nd term of the right hand side of (1.10) tends to 0 as $a \rightarrow x_{0}+0$, hence we obtain

$$
\begin{aligned}
& n J_{1}(\gamma)+J_{2}(\gamma)=2 \lim _{a \rightarrow x_{0}+0}\left[-\frac{2 \sqrt{n-a}\left\{\lambda\left(x_{1}\right)-\lambda(a)\right\}}{(1-a) \sqrt{a(n-a)^{n-1}-c}}\right. \\
& \left.+\int_{a}^{x_{1}-x(n-x)^{n-1}+c+n(n-x)^{n}\left\{\lambda\left(x_{1}\right)-\lambda(x)\right\}} \frac{\sqrt{ }(n-x)^{3}\left\{x(n-x)^{n-1}-c\right\}^{3}}{\sqrt{3}}\right] .
\end{aligned}
$$


§2. An expression of $n J_{1}(\gamma)+J_{2}(\gamma)$.

For simplicity, setting

$$
\begin{aligned}
\tilde{F}(x)=\tilde{F}\left(x, x_{1}\right):= & -x(n-x)^{n-1}+x_{1}\left(n-x_{1}\right)^{n-1} \\
& +n(n-x)^{n}\left\{\lambda\left(x_{1}\right)-\lambda(x)\right\},
\end{aligned}
$$

we have easily

$$
\tilde{F}\left(x_{0}\right)=n\left(n-x_{0}\right)^{n}\left\{\lambda\left(x_{1}\right)-\lambda\left(x_{0}\right)\right\}>0 \text { and } \tilde{F}\left(x_{1}\right)=0
$$

by Lemma 2.2 in [11]. Since we have

$$
\begin{aligned}
\tilde{F}^{\prime}(x)= & -n(1-x)(n-x)^{n-2}-n^{2}(n-x)^{n-1}\left\{\lambda\left(x_{1}\right)-\lambda(x)\right\} \\
& +n(n-x)^{n} \frac{1-x}{(n-x)^{2}}=-n^{2}(n-x)^{n-1}\left\{\lambda\left(x_{1}\right)-\lambda(x)\right\},
\end{aligned}
$$

we have

$$
\tilde{F}^{\prime}(x)<0 \text { for } x_{0} \leqq x<x_{1}, \tilde{F}^{\prime}(x)>0 \text { for } x_{1}<x<n
$$

and $\tilde{F}^{\prime}\left(x_{1}\right)=0$. Hence $\tilde{F}(x)>0$ for $x_{0} \leqq x<n, x \neq x_{1}$.

Next we have

$$
\begin{aligned}
& \frac{\sqrt{n-x}\left\{\lambda\left(x_{1}\right)-\lambda(x)\right\}}{(1-x) \sqrt{\phi(x)-c}}=\frac{\sqrt{n-x}}{(1-x) \sqrt{\phi(x)-c}} \cdot \frac{\tilde{F}(x)+\psi(x)-c}{n(n-x)^{n}} \\
= & \frac{\tilde{F}(x)}{n(1-x)(n-x)^{n-1 / 2} \sqrt{\phi(x)-c}}+\frac{\sqrt{\phi(x)-c}}{n(1-x)(n-x)^{n-1 / 2}},
\end{aligned}
$$

and

$$
\lim _{a \rightarrow x_{0}} \frac{\sqrt{\phi(a)-c}}{n(1-a)(n-a)^{n-1 / 2}}=0, \quad \lim _{a \rightarrow x_{0}} \frac{B-\psi(a)}{B-c}=1,
$$

where $B=(n-1)^{n-1}$. Hence, $(1.11)$ can be written as

$$
\begin{aligned}
n J_{1}(\gamma)+J_{2}(\gamma)=2 \lim _{a \rightarrow x_{0}}[ & -\frac{2}{n b^{2}} \cdot \frac{\tilde{F}(a)\{B-\psi(a)\}}{(1-a)(n-a)^{n-1 / 2} \sqrt{\phi(a)-c}} \\
& \left.+\int_{a}^{x_{1}} \frac{\tilde{F}(x) d x}{\sqrt{(n-x)^{3}\{\phi(x)-c\}^{3}}}\right] .
\end{aligned}
$$

Using the function $\mu(x)$ defined by (4.8) in [11]:

$$
\mu(x)= \begin{cases}\frac{B-\psi(x)}{(x-1)^{2}} & \text { for } 0<x<n, x \neq 1 \\ \frac{n(n-1)^{n-2}}{2} & \text { for } x=1,\end{cases}
$$

we define an auxiliary function 


$$
L(x):= \begin{cases}\frac{(1-x) \mu(x) \tilde{F}(x)}{(n-x)^{n-1 / 2}} & \text { for } 0<x<n, x \neq 1, \\ 0 & \text { for } x=1,\end{cases}
$$

which is real analytic in $(0, n)$, and we get

and

$$
\tilde{L}(x)>0 \text { for } x_{0} \leqq x<1 \text { and } \tilde{L}(x)<0 \text { for } 1<x<n, x \neq x_{1} \text {, }
$$

$$
\widetilde{L}(1)=\widetilde{L}\left(x_{1}\right)=\widetilde{L}^{\prime}\left(x_{1}\right)=0 .
$$

Using $\widetilde{L}(x)$, (2.1) can be written as

$$
n J_{1}(\gamma)+J_{2}(\gamma)=2 \lim _{a \rightarrow x_{0}}\left[-\frac{2}{n b^{2}} \frac{\widetilde{L}(a)}{\sqrt{\psi(a)-c}}+\int_{a}^{x_{1}} \frac{\tilde{F}(x) d x}{\sqrt{(n-x)^{3}\{\phi(x)-c\}^{3}}}\right] .
$$

We have

$$
\lim _{x \rightarrow x_{1}} \frac{\widetilde{L}(x)}{\sqrt{\psi(x)-c}}=0,
$$

since $\tilde{L}(x)$ has a zero point of order at least 2 at $x=x_{1}$, and

$$
\left(\frac{\widetilde{L}(x)}{\sqrt{\psi(x)-c}}\right)^{\prime}=\frac{2\{\phi(x)-c\} \widetilde{L}^{\prime}(x)-n(1-x)(n-x)^{n-2} \widetilde{L}(x)}{2 \sqrt{\{\phi(x)-c\}^{3}}} .
$$

Hence we have

$$
\begin{aligned}
& -\frac{2}{n b^{2}} \frac{\tilde{L}(a)}{\sqrt{\psi(a)-c}}+\int_{a}^{x_{1}} \frac{\tilde{F}(x) d x}{\sqrt{(n-x)^{3}\{\phi(x)-c\}^{3}}} \\
= & \frac{2}{n b^{2}} \int_{a}^{x_{1}}\left(\frac{\tilde{L}(x)}{\sqrt{\psi(x)-c}}\right)^{\prime} d x+\int_{a}^{x_{1}} \frac{\tilde{F}(x) d x}{\sqrt{(n-x)^{3}\{\phi(x)-c\}^{3}}} \\
= & \frac{1}{b^{2}} \int_{a}^{x_{1}} \frac{1}{\sqrt{(n-x)^{3}\{\phi(x)-c\}^{3}}}\left[b^{2} \tilde{F}(x)+\frac{2}{n}(n-x)^{3 / 2}\{\phi(x)-c\} \widetilde{L}^{\prime}(x)\right. \\
& \left.-(1-x)(n-x)^{n-1 / 2} \widetilde{L}(x)\right] d x .
\end{aligned}
$$

The expression in the brackets of the last equality can be written as

$$
\begin{aligned}
& b^{2} \tilde{F}(x)+\frac{2}{n}(n-x)^{3 / 2}\{\phi(x)-c\} \widetilde{L}^{\prime}(x)-(1-x)(n-x)^{n-1 / 2} \widetilde{L}(x) \\
& =\left\{\hat{F}(x)+\frac{2}{n}(n-x)^{3 / 2} \widetilde{L}^{\prime}(x)\right\}\{\psi(x)-c\} .
\end{aligned}
$$

By (2.1) and (2.2) we have

$$
\tilde{F}(x)+\frac{2}{n}(n-x)^{3 / 2} \widetilde{L}^{\prime}(x)
$$




$$
\begin{gathered}
=\tilde{F}(x)+\frac{2}{n} \frac{\{B-\psi(x)\} \tilde{F}(x)}{(1-x)(n-x)^{n-2}}\left[-\frac{n^{2}(n-x)^{n-1}\left\{\lambda\left(x_{1}\right)-\lambda(x)\right\}}{\tilde{F}(x)}\right. \\
\left.-\frac{n(1-x)(n-x)^{n-2}}{B-\psi(x)}+\frac{4 n-1-(2 n+1) x}{2(1-x)(n-x)}\right] \\
=-\tilde{F}(x)-\frac{2 n(n-x)\{B-\psi(x)\}\left\{\lambda\left(x_{1}\right)-\lambda(x)\right\}}{1-x} \\
+\frac{\{4 n-1-(2 n+1) x\}\{B-\psi(x)\} \tilde{F}(x)}{n(1-x)^{2}(n-x)^{n-1}} .
\end{gathered}
$$

Now, setting

$$
\begin{aligned}
\tilde{M}(x):=\tilde{F}(x) & +\frac{2 n(n-x)\{B-\phi(x)\}\left\{\lambda\left(x_{1}\right)-\lambda(x)\right\}}{1-x} \\
& -\frac{\{4 n-1-(2 n+1) x\}\{B-\psi(x)\} \tilde{F}(x)}{n(1-x)^{2}(n-x)^{n-1}},
\end{aligned}
$$

we obtain

$$
-\frac{2}{n b^{2}} \frac{\tilde{L}(a)}{\sqrt{ } \overline{\psi(a)-c}}+\int_{a}^{x_{1}} \frac{\tilde{F}(x) d x}{\sqrt{(n-x)^{3}\{\psi(x)-c\}^{3}}}=-\frac{1}{b^{2}} \int_{a}^{x_{1}} \frac{\tilde{M}(x) d x}{\sqrt{(n-x)^{3}\{\overline{\psi(x)-c)}}} .
$$

By the properties of $\tilde{F}(x)$ and (2.5), we see easily that

$$
\tilde{M}\left(x_{1}\right)=0
$$

and

$$
\begin{aligned}
\tilde{M}\left(x_{0}\right)= & n\left(n-x_{0}\right)^{n}\left\{\lambda\left(x_{1}\right)-\lambda\left(x_{0}\right)\right\}+\frac{2 n\left(n-x_{0}\right)(B-c)\left\{\lambda\left(x_{1}\right)-\lambda\left(x_{0}\right)\right\}}{1-x_{0}} \\
& -\frac{\left\{4 n-1-(2 n+1) x_{0}\right\}\left(n-x_{0}\right)(B-c)\left\{\lambda\left(x_{1}\right)-\lambda\left(x_{0}\right)\right\}}{\left(1-x_{0}\right)^{2}} \\
= & \frac{\left(n-x_{0}\right)\left\{\lambda\left(x_{1}\right)-\lambda\left(x_{0}\right)\right\}}{\left(1-x_{0}\right)^{2}}\left[n\left(1-x_{0}\right)^{2}\left(n-x_{0}\right)^{n-1}\right. \\
& \left.\quad+2 n\left(1-x_{0}\right)(B-c)-\left\{4 n-1-(2 n+1) x_{0}\right\}(B-c)\right],
\end{aligned}
$$

i. e.

$$
\begin{array}{r}
\tilde{M}\left(x_{0}\right)=\frac{\left(n-x_{0}\right)\left\{\lambda\left(x_{1}\right)-\lambda\left(x_{0}\right)\right\}}{\left(1-x_{0}\right)^{2}}\left[\left(n-x_{0}+(n-1) x_{0}{ }^{2}\right)\left(n-x_{0}\right)^{n-1}\right. \\
\left.-\left(2 n-1-x_{0}\right) B\right] .
\end{array}
$$

Since $\tilde{M}(x)$ is real analytic in $0<x<n$, we obtain finally the formula

$$
n J_{1}(\gamma)+J_{2}(\gamma)=-\frac{2}{b^{2}} \int_{x_{0}}^{x_{1}} \frac{\tilde{M}(x) d x}{\sqrt{(n-x)^{3}\left\{x(n-x)^{n-1}-c\right\}}} .
$$

As in the argument in [11], we consider a complex valued function for $\tilde{M}(x)$ by 


$$
\begin{aligned}
M\left(z, x_{1}\right)=[ & \left.-\frac{\{4 n-1-(2 n+1) z\}\{B-\psi(z)\}}{n(1-z)^{2}(n-z)^{n-1}}\right] \\
& \times\left[-\psi(z)+\phi\left(x_{1}\right)+n(n-z)^{n}\left\{\lambda\left(x_{1}\right)-\lambda(z)\right\}\right] \\
& +\frac{2 n(n-z)\{B-\phi(z)\}\left\{\lambda\left(x_{1}\right)-\lambda(z)\right\}}{1-z},
\end{aligned}
$$

which coincides with $M\left(z, x_{0}\right)$ defined in [12], $\S 1$, replaced $x_{0}$ by $x_{1}$, and $\tilde{M}(x)$ $=M\left(x, x_{1}\right)$. Hence, using the auxiliary functions

$$
\begin{aligned}
& f_{0}(z):=(2 n-1-z) B-(n-z)^{n-1}\left\{n-z+(n-1) z^{2}\right\}, \\
& f_{1}(z):=\{4 n-1-(2 n+1) z\} B-(n-z)^{n-1}\left\{n+(2 n-1) z-(n+1) z^{2}\right\},
\end{aligned}
$$

we obtain the equality

$$
\begin{aligned}
M\left(z, x_{1}\right)= & -\frac{n-z}{(1-z)^{2}} f_{0}(z)\left\{\lambda\left(x_{1}\right)-\lambda(z)\right\} \\
& +\frac{1}{n(1-z)^{2}(n-z)^{n-1}} f_{1}(z)\left\{\psi(z)-\phi\left(x_{1}\right)\right\}
\end{aligned}
$$

(see $\S 1$ in [12]).

Now, using the function $X=X_{n}(x)(0 \leqq x \leqq 1)$ defined by

$$
x(n-x)^{n-1}=X(n-X)^{n-1}, \quad 1 \leqq X \leqq n
$$

with

$$
\frac{d X}{d x}=\frac{1-x}{x(n-x)} \cdot \frac{X(n-X)}{1-X}
$$

we have

$$
\int_{x_{0}}^{x_{1}} \frac{M\left(x, x_{1}\right) d x}{\sqrt{(n-x)^{3}\left\{x(n-x)^{n-1}-c\right\}}}=\int_{x_{0}}^{1} \frac{1-x}{x(n-x) \sqrt{x(n-x)^{n-1}-c}} K\left(x, x_{1}\right) d x,
$$

where

$$
K\left(x, x_{1}\right):=\left\{\begin{array}{l}
\frac{x M\left(x, x_{1}\right)}{(1-x) \sqrt{n-x}}-\frac{X M\left(X, x_{1}\right)}{(1-X) \sqrt{n-X}} \text { for } 0<x<1 \\
0 \quad \text { for } x=1
\end{array}\right.
$$

By means of (2.11), we obtain easily

$$
\begin{aligned}
K\left(x, x_{1}\right)= & \frac{x \sqrt{n-x}}{(x-1)^{3}} f_{0}(x)\left\{\lambda\left(x_{1}\right)-\lambda(x)\right\}+\frac{x^{2} f_{1}(x)}{n(1-x)^{3} \sqrt{n-x}} \cdot \frac{\phi(x)-\psi\left(x_{1}\right)}{\psi(x)} \\
& -\frac{X \sqrt{n-X}}{(X-1)^{3}} f_{0}(X)\left\{\lambda\left(x_{1}\right)-\lambda(X)\right\}-\frac{X^{2} f_{1}(X)}{n(1-X)^{3} \sqrt{n-X}} \cdot \frac{\phi(X)-\psi\left(x_{1}\right)}{\psi(X)}
\end{aligned}
$$

and hence 


$$
\begin{aligned}
K\left(x, x_{1}\right)= & \frac{x \sqrt{n-x} f_{0}(x)}{(x-1)^{3}}\{\lambda(X)-\lambda(x)\} \\
& -\left[\frac{X \sqrt{n-X} f_{0}(X)}{(X-1)^{3}}-\frac{x \sqrt{n-x} f_{0}(x)}{(x-1)^{3}}\right]\left\{\lambda\left(x_{1}\right)-\lambda(X)\right\} \\
& -\left[\frac{X^{2} f_{1}(X)}{(1-X)^{3} \sqrt{n-X}}-\frac{x^{2} f_{1}(x)}{(1-x)^{3} \sqrt{n-x}}\right] \frac{\left\{\phi(x)-\psi\left(x_{1}\right)\right\}}{n \psi(x)} .
\end{aligned}
$$

REMARK. The first term and the functions in the two pairs of brackets are all positive by Lemma 2.2 in [11] and Proposition 2 and Proposition 4 in [12].

\section{$\S 3$. An expression of $\partial \Omega(\tau, n) / \partial n$ and some constants.}

LEMMA 3.1. We have the formula:

$$
\frac{\partial \Omega(\tau, n)}{\partial n}=-\frac{\sqrt{c}}{2 b^{2} \sqrt{n}} \int_{x_{0}}^{1} \frac{(1-x) W\left(x, x_{1}\right) d x}{x(n-x) \sqrt{x(n-x)^{n-1}-c}}
$$

where $c=x_{1}\left(n-x_{1}\right)^{n-1}, b=\sqrt{B-c}, B=(n-1)^{n-1}$ and

$$
\begin{aligned}
W(x, & \left.x_{1}\right):=\frac{x \sqrt{n-x} f_{0}(x)}{(x-1)^{3}}\{\lambda(X)-\lambda(x)\} \\
+ & {\left[\frac{X \sqrt{n-X} f_{0}(X)}{(X-1)^{3}}-\frac{x \sqrt{n-x} f_{0}(x)}{(x-1)^{3}}\right] \cdot\left\{\frac{n}{n-1} \frac{\left(x_{1}-1\right)^{2}}{x_{1}\left(n-x_{1}\right)}-\lambda\left(x_{1}\right)+\lambda(X)\right\} } \\
& -\left[\frac{X^{2} f_{1}(X)}{(1-X)^{3} \sqrt{n-X}}-\frac{x^{2} f_{1}(x)}{(1-x)^{3} \sqrt{n-x}}\right] \cdot \frac{\psi(x)-\psi\left(x_{1}\right)}{n \psi(x)} .
\end{aligned}
$$

Proof. From (1.9), (2.8) and (2.14), we have

$$
\begin{aligned}
\frac{\partial \Omega(\tau, n)}{\partial n}= & -\frac{\sqrt{c}}{2 b^{2} \sqrt{n}} \int_{x_{0}}^{1} \frac{1-x}{x(n-x) \sqrt{x(n-x)^{n-1}-c}} K\left(x, x_{1}\right) d x \\
& +\frac{n \sqrt{n}\left(x_{1}-1\right)^{2}\left(n-x_{1}\right)^{n-2}}{4(n-1) \sqrt{c}} J_{3}(\gamma) .
\end{aligned}
$$

On the other hand, by (6) in Appendix in [12] we have

$$
J_{3}(\gamma)=-\frac{2}{n b^{2}} \int_{x_{0}}^{1} \frac{1-x}{x(n-x) \sqrt{x(n-x)^{n-1}-c}}\left[\frac{X \sqrt{n-X} f_{0}(X)}{(X-1)^{3}}-\frac{x \sqrt{n-x} f_{0}(x)}{(x-1)^{3}}\right] d x .
$$

Hence, we obtain

$$
\begin{aligned}
\frac{\partial \Omega(\tau, n)}{\partial n}=- & \frac{\sqrt{c}}{2 b^{2} \sqrt{n}} \int_{x_{0}}^{1} \frac{1-x}{x(n-x) \sqrt{x(n-x)^{n-1}}-c}\left[K\left(x, x_{1}\right)\right. \\
& \left.+\frac{n}{n-1} \frac{\left(x_{1}-1\right)^{2}\left(n-x_{1}\right)^{n-2}}{c}\left\{\frac{X \sqrt{n-X} f_{0}(X)}{(X-1)^{3}}-\frac{x \sqrt{n-x} f_{0}(x)}{(x-1)^{3}}\right\}\right] d x .
\end{aligned}
$$

By (2.16), we have 


$$
\begin{aligned}
& K\left(x, x_{1}\right)+\frac{n}{n-1} \frac{\left(x_{1}-1\right)^{2}\left(n-x_{1}\right)^{n-2}}{c}\left\{\frac{X \sqrt{n-X} f_{0}(X)}{(X-1)^{3}}-\frac{x \sqrt{n-x} f_{0}(x)}{(x-1)^{3}}\right\} \\
& =\frac{x \sqrt{n-x} f_{0}(x)}{(x-1)^{3}}\{\lambda(X)-\lambda(x)\} \\
& +\left[\frac{X \sqrt{n-X} f_{0}(X)}{(X-1)^{3}}-\frac{x \sqrt{n-x} f_{0}(x)}{(x-1)^{3}}\right] \cdot\left\{\frac{n}{n-1} \frac{\left(x_{1}-1\right)^{2}}{x_{1}\left(n-x_{1}\right)}-\lambda\left(x_{1}\right)+\lambda(X)\right\} \\
& -\left[\frac{X^{2} f_{1}(X)}{(1-X)^{3} \sqrt{n-X}}-\frac{x^{2} f_{1}(x)}{(1-x)^{3} \sqrt{n-x}}\right] \cdot \frac{\phi(x)-\psi\left(x_{1}\right)}{n \psi(x)}=W\left(x, x_{1}\right)
\end{aligned}
$$

Q.E. D.

Lemma 3.2. On $W\left(x, x_{1}\right)$, we have

$$
W(x, X(x))>0 \text { and } \lim _{x_{1} \rightarrow n} W\left(x, x_{1}\right)=+\infty \quad \text { for } 0<x<1 .
$$

Proof. From (3.1) we get easily

$$
\begin{aligned}
W(x, X(x))= & \frac{x \sqrt{n-x} f_{0}(x)}{(x-1)^{3}}\{\lambda(X)-\lambda(x)\} \\
& +\left[\frac{X \sqrt{n-X} f_{0}(X)}{(X-1)^{2}}-\frac{x \sqrt{n-x} f_{0}(x)}{(x-1)^{3}}\right] \cdot \frac{n}{n-1} \frac{(X-1)^{2}}{X(n-X)}>0,
\end{aligned}
$$

because $\frac{f_{0}(x)}{(x-1)^{3}}>0$ and $\frac{X \sqrt{n-X} f_{0}(X)}{(X-1)^{3}}-\frac{x \sqrt{n-x} f_{0}(x)}{(x-1)^{3}}>0$ by Lemma 2.1 and Proposition 2 in [12], and $\lambda(X)-\lambda(x)>0$ by Lemma 2.2 in [11].

Next, we shall show that the 2 nd factor of the 2 nd term of (3.1) diverges to $+\infty$ as $x_{1} \rightarrow n-0$. Since we have

$$
\frac{n}{n-1} \frac{\left(x_{1}-1\right)^{2}}{x_{1}\left(n-x_{1}\right)}-\lambda\left(x_{1}\right)=\frac{n\left(x_{1}-1\right)^{2}-(n-1) x_{1}\left\{\left(n-x_{1}\right) \log (n-x)+n-1\right\}}{(n-1) x_{1}\left(n-x_{1}\right)},
$$

we obtain

$$
\begin{aligned}
& \lim _{x_{1} \rightarrow n}\left[\frac{n\left(x_{1}-1\right)^{2}}{(n-1) x_{1}\left(n-x_{1}\right)}-\lambda\left(x_{1}\right)\right] \\
& =\frac{1}{n(n-1)} \lim _{x_{1} \rightarrow n} \frac{n\left(x_{1}-1\right)^{2}-(n-1) x_{1}\left\{\left(n-x_{1}\right) \log \left(n-x_{1}\right)+n-1\right\}}{n-x_{1}} \\
& =\frac{1}{n(n-1)} \lim _{x_{1} \rightarrow n} \frac{2 n\left(x_{1}-1\right)-(n-1)\left\{\left(n-2 x_{1}\right) \log \left(n-x_{1}\right)-x_{1}+n-1\right\}}{-1} \\
& =-\frac{1}{n(n-1)} \lim _{x_{1} \rightarrow n}\left[2 n(n-1)+n-1+n(n-1) \log \left(n-x_{1}\right)\right]=+\infty,
\end{aligned}
$$

from which we obtain immediately

$$
\lim _{x_{1 \rightarrow n}} W\left(x, x_{1}\right)=+\infty .
$$


LEMMA 3.3. We have

$$
\lim _{x \rightarrow 1-0} W\left(x, x_{1}\right)=0 \quad \text { for } 1<x_{1}<n .
$$

Proof. Since we have $\lim _{x \rightarrow 1} X(x)=1$, we obtain this assertion by Lemma 2.2 and Lemma 4.2 in [12].

Now, we consider the increasing ratio of the function $W\left(x, x_{1}\right)$ defined above in the domain: $0<x<1, X_{n}(x) \leqq x_{1}<n$, with respect to $x_{1}$. Using the auxiliary functions $F_{0}(x)$ and $F_{1}(x)$ defined by (2.3) and (4.4) in [12] respectively as follow:

$$
F_{0}(x)= \begin{cases}(n-x)^{-n+3 / 2}(x-1)^{-3} f_{0}(x) & \text { for } 0 \leqq x<n, x \neq 1 \\ n(2 n-1) / 6 \sqrt{n-1} & \text { for } x=1\end{cases}
$$

and

$$
F_{1}(x)= \begin{cases}(n-x)^{-2 n+3 / 2}(1-x)^{-3} f_{1}(x) & \text { for } 0 \leqq x<n, x \neq 1 \\ n(4 n+1) / 6(n-1)^{n+1 / 2} & \text { for } x=1,\end{cases}
$$

we obtain the following

LEMMA 3.4. We have $\frac{\partial W\left(x, x_{1}\right)}{\partial x_{1}} \geqq 0$, if and only if

$$
\frac{F_{0}(X)-F_{0}(x)}{F_{1}(X)-F_{1}(x)} \geqq \frac{(n-1) x_{1}^{2}\left(n-x_{1}\right)^{n-1}}{n+(n-1) x_{1}},
$$

where $X(x)<x_{1}<n$.

Proof. We obtain from (3.1), (3.2) and (3.3)

$$
\begin{aligned}
\frac{W\left(x, x_{1}\right)}{\phi(x)}= & F_{0}(x)\{\lambda(X)-\lambda(x)\} \\
& +\left[F_{0}(X)-F_{0}(x)\right]\left\{\frac{n}{n-1}-\frac{\left(x_{1}-1\right)^{2}}{x_{1}\left(n-x_{1}\right)}-\lambda\left(x_{1}\right)+\lambda(X)\right\} \\
& -\frac{1}{n}\left[F_{1}(X)-F_{1}(x)\right]\left\{\phi(x)-\phi\left(x_{1}\right)\right\}
\end{aligned}
$$

and

$$
\begin{aligned}
\frac{1}{\phi(x)} \frac{\partial W\left(x, x_{1}\right)}{\partial x_{1}}= & {\left[F_{0}(X)-F_{0}(x)\right]\left\{\frac{n}{n-1} \frac{\left(x_{1}-1\right)\left\{n+(n-2) x_{1}\right\}}{x_{1}^{2}\left(n-x_{1}\right)^{2}}-\frac{x_{1}-1}{\left(n-x_{1}\right)^{2}}\right\} } \\
& -\left[F_{1}(X)-F_{1}(x)\right]\left(x_{1}-1\right)\left(n-x_{1}\right)^{n-2} \\
= & {\left[F_{0}(X)-F_{0}(x)\right] \frac{\left(x_{1}-1\right)\left\{n+(n-1) x_{1}\right\}}{(n-1) x_{1}^{2}\left(n-x_{1}\right)} } \\
& -\left[F_{1}(X)-F_{1}(x)\right]\left(x_{1}-1\right)\left(n-x_{1}\right)^{n-2} .
\end{aligned}
$$


Since $F_{0}(X)-F_{0}(x)$ and $F_{1}(X)-F_{1}(x)$ are positive by Proposition 2 and Proposition 4 in [12], we obtain immediately from the last equality the statement in this lemma.

Q.E. D.

LEMMA 3.5. The function $\frac{x^{2}(n-x)^{n-1}}{n+(n-1) x}$ takes its maxımum for $0 \leqq x \leqq n$ at $\beta=\beta(n)$ given by

$$
\beta:=\left(\sqrt{2 n^{2}-2 n+1}-1\right) /(n-1),
$$

which is increasing with respect to $n$ and tends to $\sqrt{2}$ as $n \rightarrow \infty$.

Proof. We have

$$
\frac{d}{d x} \frac{x^{2}(n-x)^{n-1}}{n+(n-1) x}=-\frac{n x(n-x)^{n-2}\left\{(n-1) x^{2}+2 x-2 n\right\}}{\{n+(n-1) x\}^{2}}
$$

from which we see that the given function is increasing in $[0, \beta]$ and decreasing in $[\beta, n]$, where $\beta$ is the positive root of the quadratic equation: $(n-1) x^{2}+2 x-2 n$ $=0$ given by (3.5). We get easily

$$
1<\beta<2 \text {. }
$$

Next, we have

$$
\frac{d \beta(n)}{d n}=\frac{\sqrt{2 n^{2}-2 n+1}-n}{(n-1)^{2} \sqrt{2 n^{2}-2 n+1}}>0 \text { for } n>1,
$$

hence $\beta(n)$ is increasing with respect to $n$. It is evident that

$$
\beta(n) \uparrow \sqrt{2} \text { as } n \rightarrow \infty . \quad \text { Q. E. D. }
$$

LEMMA 3.6. When $n>1$, we have

$$
\sqrt{\frac{n}{n+1}} \sqrt{2}<\beta(n)<\sqrt{2}
$$

Proof. Since we have

$$
\begin{aligned}
(n-1) x^{2}+2 x-\left.2 n\right|_{x=\sqrt{\frac{2 n}{n+1}}} & =\frac{2 n(n-1)}{n+1}+2 \sqrt{\frac{2 n}{n+1}}-2 n \\
& =\frac{2 \sqrt{2 n}}{n+1}(\sqrt{n+1}-\sqrt{2 n})<0 . \quad \text { Q. E. D. }
\end{aligned}
$$

LEMMA 3.7. The constant $\nu=\nu(n)$ defined by

$$
\nu:=\frac{(n-1) \beta(n)}{n+(n-1) \beta(n)}
$$

is increasing with respect to $n$ for $n>1$ and tends to $2-\sqrt{2}$ as $n \rightarrow \infty$. Using $\nu$ we have 


$$
\left.\frac{(n-1) x^{2}(n-x)^{n-1}}{n+(n-1) x}\right|_{x=\beta}=\nu \phi(\beta) .
$$

Proof. From (3.5) and (3.9) we obtain

$$
\nu=\frac{\sqrt{2 n^{2}-2 n+1}-1}{\sqrt{ } 2 n^{2}-2 n+1+n-1}=\frac{2 n-1-\sqrt{2 n^{2}-2 n+1}}{n}
$$

and

$$
\frac{d \nu(n)}{d n}=\frac{\sqrt{2 n^{2}-2 n+1}-(n-1)}{n^{2} \sqrt{2 n^{2}-2 n+1}}>0 .
$$

The rest of the statement of this lemma is evident.

Q.E. D.

\section{$\S 4$. Auxiliary function $F_{0}(x)-\nu \phi(\beta) F_{1}(x)$.}

The author conjectured at first the function $W\left(x, x_{1}\right), X(x) \leqq x_{1}<n$, would be positive and has made efforts to prove it as shown in $\S \S 4,5,6$. But he found it was false. Being based on the facts described in these sections, especially Lemma 6.5, he will devise the expression of the main integral

$$
\frac{\partial \Omega(\tau, n)}{\partial n}=-\frac{c}{2 b^{2} \sqrt{ } n} \int_{x_{0}}^{1} \frac{(1-x) W\left(x, x_{1}\right) d x}{x(n-x) \sqrt{x(n-x)^{n-1}-c}}
$$

as the one to appear in $\S 7$. Therefore, $\S 7$ should be followed logically to the end of $\S 3$. In order to show why he has to introduce the auxiliary function $V\left(x, x_{1}\right)$ by $(7.10)$ in place of $W\left(x, x_{1}\right)$, he will describe them in brief, since these results in $\S \S 4,5,6$ may be used in the arguments after $\S 7$ in the future.

First of all, noticing Lemma 3.4, Lemma 3.5 and Lemma 3.7, we set an auxiliary function $G(x)$ for each $n$ by

$$
G(x):=F_{0}(x)-\nu \psi(\beta) F_{1}(x) \text { for } 0 \leqq x<n .
$$

LEMMA 4.1. We have $G^{\prime}(x) \geqq 0$ if and only if

$$
(n-x)^{n} F_{2}(x)-\nu \phi(\beta) F_{3}(x) \geqq 0,
$$

where

$$
\begin{aligned}
F_{2}(x)= & -\left\{(2 n+1) x^{2}-2\left(2 n^{2}+5 n-4\right) x+16 n^{2}-16 n+3\right\} B \\
& +(n-x)^{n-1}\left\{-(n-1) x^{3}+\left(2 n^{2}-7 n+8\right) x^{2}+(n-3)(4 n-1) x+3 n(2 n-1)\right\}
\end{aligned}
$$

and

$$
\begin{aligned}
& F_{3}(x)=\left\{3(2 n-1)(6 n-1)-2\left(16 n^{2}+3 n-4\right) x+(2 n+1)(4 n+1) x^{2}\right\} B \\
& -(n-x)^{n-1}\left\{3 n(4 n-1)+3\left(2 n^{2}-7 n+1\right) x-\left(8 n^{2}+3 n-8\right) x^{2}+(n+1)(2 n+1) x^{3}\right\},
\end{aligned}
$$

which have been introduced in [12] by (2.6) and (4.8) respectively.

Proof. By means of (2.7) and (4.9) in [12], we obtain 


$$
\begin{aligned}
G^{\prime}(x) & =F_{0}^{\prime}(x)-\nu \phi(\beta) F_{1}^{\prime}(x) \\
& =\frac{1}{2}(x-1)^{-4}(n-x)^{-2 n+1 / 2}\left\{(n-x)^{n} F_{2}(x)-\nu \phi(\beta) F_{3}(x)\right\}
\end{aligned}
$$

for $0 \leqq x<n, x \neq 1$, from which we obtain immediately this lemma.

Q. E. D.

Now, by Lemma $4.1, G^{\prime}(x) \geqq 0$ at $x(0 \leqq x<n, x \neq 1)$ if and only if

$$
(n-x)^{n} F_{2}(x)-\nu \phi(\beta) F_{3}(x) \geqq 0 \text {. }
$$

Since $F_{2}(x)$ is positive for $0 \leqq x \leqq n, x \neq 1$ by Proposition 1 in [12], we see that for $0 \leqq x \leqq \beta$ this inequality is followed from the inequality:

$$
(n-\beta)^{n} F_{2}(x)-\nu \psi(\beta) F_{3}(x) \geqq 0,
$$

which is equivalent to

$$
(n-\beta) F_{2}(x)-\nu \beta F_{3}(x) \geqq 0
$$

Now using the auxiliary polynomials in $x$ :

$$
\begin{aligned}
& P_{2}(x)=(2 n+1) x^{2}-2\left(2 n^{2}+5 n-4\right) x+16 n^{2}-16 n+3, \\
& P_{3}(x)=-(n-1) x^{3}+\left(2 n^{2}-7 n+8\right) x^{2}+\left(4 n^{2}-13 n+3\right) x+3 n(2 n-1), \\
& \tilde{P}_{2}(x)=3(2 n-1)(6 n-1)-2\left(16 n^{2}+3 n-4\right) x+(2 n+1)(4 n+1) x^{2}, \\
& \widetilde{P}_{3}(x)=3 n(4 n-1)+3\left(2 n^{2}-7 n+1\right) x-\left(8 n^{2}+3 n-8\right) x^{2}+(n+1)(2 n+1) x^{3},
\end{aligned}
$$

which have been introduced in $[12]$, we can write $F_{2}(x)$ and $F_{3}(x)$ as

$$
F_{2}(x)=-P_{2}(x) B+(n-x)^{n-1} P_{3}(x)
$$

and

$$
F_{3}(x)=\tilde{P}_{2}(x) B-(n-x)^{n-1} \tilde{P}_{3}(x)
$$

Setting

$$
\left\{\begin{array}{l}
S_{2}(x):=(n-\beta) P_{2}(x)+\nu \beta \widetilde{P}_{2}(x), \\
S_{3}(x):-(n-\beta) P_{3}(x)+\nu \beta \widetilde{P}_{3}(x),
\end{array}\right.
$$

we have

$$
(n-\beta) F_{2}(x)-\nu \beta F_{3}(x)=(n-x)^{n-1} S_{3}(x)-S_{2}(x) B
$$

Since $\frac{8(n-1)}{2 n+1} \geqq \frac{8}{5}$ for $n \geqq 2$, it must be $\beta<\gamma_{0}$ by Lemma 3.5 and Lemma 3.3 in [12], where $\gamma_{0}$ is the smaller root of the quadratic equation in $x: P_{2}(x)=0$. Hence we have $P_{2}(x)>0$ for $0 \leqq x \leqq \beta$. By Lemma 5.1 in [12], we have $\widetilde{P}_{2}(x)>0$ for $-\infty<x<+\infty$. Therefore, the condition (4.4) is equivalent to 


$$
\frac{(n-x)^{n-1} S_{3}(x)}{S_{2}(x)} \geqq B \quad \text { for } 0 \leqq x \leqq \beta .
$$

In the following we compute the derivative of the left hand side of (4.12). We have first

$$
\frac{d}{d x} \frac{(n-x)^{n-1} S_{3}(x)}{S_{2}(x)}=\frac{(n-x)^{n-2}}{S_{2}{ }^{2}}\left[-(n-x) S_{2}{ }^{\prime} S_{3}-S_{2} T_{3}\right]
$$

where

$$
T_{3}=-(n-x) S_{3}{ }^{\prime}+(n-1) S_{3}=(n-\beta) Q_{3}(x)+\nu \beta \tilde{Q}_{3}(x),
$$

and

$$
Q_{3}(x)=-(n-x) P_{3}{ }^{\prime}(x)+(n-1) P_{3}(x), \quad \tilde{Q}_{3}(x)=-(n-x) \tilde{P}_{3}{ }^{\prime}(x)+(n-1) \tilde{P}_{3}(x)
$$

are given explicitly by (3.5) and (5.4) in [12] as follow

$$
\begin{aligned}
Q_{3}(x)= & 2 n^{2}(n+2)+n(n-13) x+2\left(n^{3}-n^{2}-n+4\right) x^{2}-(n-1)(n+2) x^{3}, \\
\tilde{Q}_{3}(x)= & 6 n^{2}(n+1)+n\left(22 n^{2}-15 n-13\right) x-2(n+1)^{2}(7 n-4) x^{2} \\
& +(n+1)(n+2)(2 n+1) x^{3} .
\end{aligned}
$$

Hence, we have

$$
\begin{aligned}
-(n-x) S_{2}{ }^{\prime} S_{3} & -S_{2} T_{3} \\
= & -(n-x)\left\{(n-\beta) P_{2}{ }^{\prime}+\nu \beta \widetilde{P}_{2}{ }^{\prime}\right\}\left\{(n-\beta) P_{3}+\nu \beta \tilde{P}_{3}\right\} \\
& -\left\{(n-\beta) P_{2}+\nu \beta \widetilde{P}_{2}\right\}\left\{(n-\beta) Q_{3}+\nu \beta \tilde{Q}_{3}\right\} \\
= & -(n-\beta)^{2}\left\{(n-x) P_{2}{ }^{\prime} P_{3}+P_{2} Q_{3}\right\} \\
& -(n-\beta) \nu \beta\left\{(n-x)\left(P_{2}{ }^{\prime} \tilde{P}_{3}+\tilde{P}_{2}{ }^{\prime} P_{3}\right)+P_{2} \tilde{Q}_{3}+\tilde{P}_{2} Q_{3}\right\} \\
& -\nu^{2} \beta^{2}\left\{(n-x) \widetilde{P}_{2}{ }^{\prime} \tilde{P}_{3}+\tilde{P}_{2} \tilde{Q}_{3}\right\}
\end{aligned}
$$

and by means of the computations done for (3.7) and (5.6) in [12]

$$
\begin{aligned}
& (n-x) P_{2}^{\prime}(x) P_{3}(x)+P_{2}(x) Q_{3}(x)=n(n-1)(1-x)^{3} Q_{2}(x), \\
& (n-x) \tilde{P}_{2}^{\prime}(x) \widetilde{P}_{3}(x)+\tilde{P}_{2}(x) \tilde{Q}_{3}(x)=-n(1-x)^{3} \tilde{Q}_{2}(x),
\end{aligned}
$$

where $Q_{2}(x)$ and $\tilde{Q}_{2}(x)$ are given explicitly by (3.6) and (5.5) in [12] as follow

$$
\begin{aligned}
& Q_{2}(x)=4 n\left(2 n^{2}-2 n+3\right)-\left(8 n^{2}-2 n+9\right) x+(2 n+1) x^{2}, \\
& \tilde{Q}_{2}(x)=6 n\left(28 n^{3}-16 n^{2}+2 n+1\right)-3(2 n+1)\left(16 n^{3}+10 n^{2}-9 n+3\right) x \\
& \quad+(n+1)(2 n+1)^{2}(4 n+1) x^{2} .
\end{aligned}
$$

Next, by (4.5) (4.8), (4.13) and (4.14) we have 


$$
\begin{aligned}
& (n-x)\left\{P_{2}{ }^{\prime}(x) \tilde{P}_{3}(x)+\tilde{P}_{2}{ }^{\prime}(x) P_{3}(x)\right\}+P_{2}(x) \tilde{Q}_{3}(x)+\tilde{P}_{2}(x) Q_{3}(x) \\
& =2 n(1-x)^{3}\left\{-3 n\left(12 n^{3}-8 n^{2}-4 n+3\right)-\left(4 n^{4}-46 n^{3}+23 n^{2}+10 n-9\right) x\right. \\
& \left.\quad+(2 n+1)\left(n^{2}-3 n-1\right) x^{2}\right\} .
\end{aligned}
$$

Thus, we can put

$$
-(n-x) S_{2}{ }^{\prime}(x) S_{3}(x)-S_{2}(x) T_{3}(x)=n(x-1)^{3}\left\{a_{n, 0}-a_{n, 1} x-a_{n, 2} x^{2}\right\},
$$

where we set

$$
\begin{aligned}
a_{n, 0}:= & n\left\{4(n-\beta)^{2}(n-1)\left(2 n^{2}-2 n+3\right)\right. \\
& \left.-6(n-\beta) \nu \beta\left(12 n^{3}-8 n^{2}-4 n+3\right)-6 \nu^{2} \beta^{2}\left(28 n^{3}-16 n^{2}+2 n+1\right)\right\}, \\
a_{n, 1}:= & (n-\beta)^{2}(n-1)\left(8 n^{2}-2 n+9\right) \\
& +2(n-\beta) \nu \beta\left(4 n^{4}-46 n^{3}+23 n^{2}+10 n-9\right) \\
& -3 \nu^{2} \beta^{2}(2 n+1)\left(16 n^{3}+10 n^{2}-9 n+3\right), \\
a_{n, 2}:= & -(n-\beta)^{2}(n-1)(2 n+1)-2(n-\beta) \nu \beta(2 n+1)\left(n^{2}-3 n-1\right) \\
& +\nu^{2} \beta^{2}(n+1)(2 n+1)^{2}(4 n+1) .
\end{aligned}
$$

LEMMA $4.2 . \quad a_{n, 2}>0$ for $n \geqq 4$.

Proof. By Lemma 3.5 and Lemma 3.7, $\nu(n) \beta(n)$ is increasing with respect to $n$, and from (3.5) and (3.11) we have

$$
\nu(n) \beta(n)=\frac{2\left\{\sqrt{2 n^{2}-2 n+1}-n\right\}}{n-1},
$$

from which we obtain $\frac{2}{3} \leqq \nu(n) \beta(n)<2(\sqrt{2}-1)$ for $4 \leqq n<\infty$. Thus, for $n \geqq 4$ we obtain from (4.23)

$$
\begin{aligned}
a_{n, 2} & >-(n-1)^{3}(2 n+1)-2(n-1)(2 n+1)\left(n^{2}-3 n-1\right)+\frac{4}{9}(n+1)(2 n+1)^{2}(4 n+1) \\
& =\frac{1}{9}\left(10 n^{4}+315 n^{3}+85 n^{2}-45 n-5\right)>0,
\end{aligned}
$$

since $n-\beta<n-1,2(\sqrt{2}-1)<1$ and $n^{2}-3 n-1>0$.

Q.E. D.

LEMMA 4.3. The function $\sigma=\sigma(n)$ of $n$ given by

$$
\sigma=\sigma(n):=(n-\beta(n)) / n
$$

is increasing for $n>1$. 
Proof. From (3.5) we have

$$
\sigma=\frac{n^{2}-n+1-\sqrt{2 n^{2}-2 n+1}}{n(n-1)}=\frac{t+1-\sqrt{2 t+1}}{t}, \text { where } t=n(n-1) .
$$

Hence we obtain

$$
\frac{d \sigma}{d n}=\frac{d \sigma}{d t} \frac{d t}{d n}=\frac{t+1-\sqrt{2 t+1}}{t^{2} \sqrt{ } 2 t+1} \cdot(2 n-1)>0 \text { for } n>1,
$$

which implies this lemma.

Q.E.D.

LEMMA 4.4. $a_{n, 0}>0$ for $n \geqq 11$.

Proof. From (4.21) and (4.25) we obtain

$$
\begin{aligned}
\frac{1}{n} a_{n, 0}= & 4 \sigma^{2}\left(2 n^{5}-4 n^{4}+5 n^{3}-3 n^{2}\right) \\
& -6 \nu \beta \sigma\left(12 n^{4}-8 n^{3}-4 n^{2}+3 n\right)-6 \nu^{2} \beta^{2}\left(28 n^{3}-16 n^{2}+2 n+1\right) .
\end{aligned}
$$

Using Lemma 3.5, Lemma 3.7 and Lemma 4.3 and dividing into the two cases: $13 \leqq n$ and $11 \leqq n<13$, we shall prove this lemma.

Case I: $13 \leqq n$. Since we have

$$
\begin{aligned}
& 4 \sigma^{2} \geqq 4(\sigma(13))^{2}=3.189804 \cdots>3.189, \\
& 6 \nu \beta \sigma<\lim _{n \rightarrow \infty} 6 \nu \beta \sigma=12 \sqrt{2}-12=4.970562 \cdots<4.971, \\
& 6 \nu^{2} \beta^{2}<\lim _{n \rightarrow \infty} 6 \nu^{2} \beta^{2}=24(3-2 \sqrt{2})=4.117749 \cdots<4.118,
\end{aligned}
$$

we obtain

$$
\begin{aligned}
\frac{1}{n} a_{n, 0}> & 3.189\left(2 n^{5}-4 n^{4}+5 n^{3}-3 n^{2}\right)-4.971\left(12 n^{4}-8 n^{3}-4 n^{2}+3 n\right) \\
& -4.118\left(28 n^{3}-16 n^{2}+2 n+1\right) \\
= & 6.378 n^{5}-72.408 n^{4}-59.591 n^{3}+76.205 n^{2}-23.149 n-4.118>0
\end{aligned}
$$

for $n \geqq 13$.

Case II : $11 \leqq n<13$. Since we have

we obtain

$$
\begin{aligned}
& 4 \sigma^{2} \geqq 4 \sigma^{2}(11)=3.055118 \cdots>3.055, \\
& 6 \nu \beta \sigma<6(\nu \beta \sigma)(13)=4.189788 \cdots<4.190, \\
& 6 \nu^{2} \beta^{2}<6\left(\nu^{2} \beta^{2}\right)(13)=3.668840 \cdots<3.669,
\end{aligned}
$$

$$
\frac{1}{n} a_{n, 0}>3.055\left(2 n^{5}-4 n^{4}+5 n^{3}-3 n^{2}\right)-4.190\left(12 n^{4}-8 n^{3}-4 n^{2}+3 n\right)
$$




$$
\begin{aligned}
& -3.669\left(28 n^{3}-16 n^{2}+2 n+1\right) \\
= & 6.11 n^{5}-62.5 n^{4}-53.937 n^{3}+66.299 n^{2}-19.908 n-3.669>0
\end{aligned}
$$

for $11 \leqq n<13$.

Q.E.D.

LEMMA $4.5 . \quad a_{n, 0}<0$ for $8 \leqq n \leqq 10$.

Proof. Since we have

we obtain

$$
\begin{aligned}
& 4 \sigma^{2} \leqq 4 \sigma^{2}(10)=2.969600 \cdots<2.970, \\
& 6 \nu \beta \sigma \geqq 6(\nu \beta \sigma)(8)=3.733453 \cdots>3.733, \\
& 6 \nu^{2} \beta^{2}>6\left(\nu^{2} \beta^{2}\right)(8)=3.388245 \cdots>3.388,
\end{aligned}
$$

$$
\begin{aligned}
\frac{1}{n} a_{n .0}< & 2.970\left(2 n^{5}-4 n^{4}+5 n^{3}-3 n^{2}\right)-3.733\left(12 n^{4}-8 n^{3}-4 n^{2}+3 n\right) \\
& -3.388\left(28 n^{3}-16 n^{2}+2 n+1\right) \\
= & 5.94 n^{5}-56.676 n^{5}-50.15 n^{3}+60.23 n^{2}-17.975 n-3.388<0
\end{aligned}
$$

for $8 \leqq n \leqq 10$.

Q.E.D.

Remark. By a computation we see that $a_{n, 0}$ change its sign at a point in the interval $10.6<n<10.7$, i. e. $n=10.69931 \cdots$.

LEMMA 4.6. $a_{n, 1}>0$ for $n \geqq 12$.

Proof. From (4.22) and (4.25) we obtain

$$
\begin{aligned}
a_{n, 1}= & \sigma^{2}\left(8 n^{5}-10 n^{4}+11 n^{3}-9 n^{2}\right) \\
& +2 \nu \beta \sigma\left(4 n^{5}-46 n^{4}+23 n^{3}+10 n^{2}-9 n\right)-3 \nu^{2} \beta^{2}\left(32 n^{4}+36 n^{3}-8 n^{2}-3 n+3\right) .
\end{aligned}
$$

Since we have for $n \geqq 12$

$$
\begin{aligned}
& \sigma^{2} \geqq \sigma^{2}(12)=0.781900 \cdots>0.781, \\
& 2 \nu \beta \sigma>2(\nu \beta \sigma)(12)=1.375838 \cdots>1.375, \\
& 3 \nu^{2} \beta^{2}<\lim _{n \rightarrow \infty} 3 \nu^{2} \beta^{2}=12(3-2 \sqrt{2})=2.058874 \cdots<2.059,
\end{aligned}
$$

we obtain

$$
\begin{aligned}
a_{n, 1}> & 0.781\left(8 n^{5}-10 n^{4}+11 n^{3}-9 n^{2}\right)+1.375\left(4 n^{5}-46 n^{4}+23 n^{3}+10 n^{2}-9 n\right) \\
& -2.059\left(32 n^{4}+36 n^{3}-8 n^{2}-3 n+3\right) \\
= & 11.748 n^{5}-136.948 n^{4}-33.908 n^{3}+23.193 n^{2}-6.198 n-6.177>0
\end{aligned}
$$


for $n \geqq 12$.

Q.E.D.

LEMMA 4.7. $a_{n, 1}<0$ for $9 \leqq n \leqq 11$.

Proof. Since we have

we obtain

$$
\begin{aligned}
& \sigma^{2} \leqq \sigma^{2}(11)=0.763779 \cdots<0.764 \\
& 2 \nu \beta \sigma \leqq 2(\nu \beta \sigma)(11)=1.351492 \cdots<1.352, \\
& 3 \nu^{2} \beta^{2} \geqq 3 \nu^{2} \beta^{2}(9)=1.734618 \cdots>1.734
\end{aligned}
$$

$$
\begin{aligned}
a_{n, 1}< & 0.764\left(8 n^{5}-10 n^{4}+11 n^{3}-9 n^{2}\right)+1.352\left(4 n^{5}-46 n^{4}+23 n^{3}+10 n^{2}-9 n\right) \\
& -1.734\left(32 n^{4}+36 n^{3}-8 n^{2}-3 n+3\right) \\
= & 11.52 n^{5}-125.32 n^{4}-22.924 n^{3}+20.516 n^{2}-6.966 n-5.202<0
\end{aligned}
$$

for $9 \leqq n \leqq 11$.

Q.E. D.

Remark. By an analogous argument to the proof of Lemma 4.7, we can prove that $a_{n, 1}<0$ for $2 \leqq n<9$ and see that $a_{n, 1}$ changes its sign at a point in the interval $11.2<n<11.3$, i. e. $n=11.21186 \cdots$.

\section{§5. Quadratic polynomial $a_{n, 0}-a_{n, 1} x-a_{n, 2} x^{2}$.}

In this section, setting

$$
Q_{n, 2}^{*}(x):=a_{n, 0}-a_{n, 1} x-a_{n, 2} x^{2}
$$

we shall investigate its sign in the interval $X^{-1}(\beta) \leqq x \leqq \beta$, when $n \geqq 11$.

First we obtain from (4.23) and (4.25)

$$
\begin{aligned}
a_{n, 2}= & -\sigma^{2}\left(2 n^{4}-n^{3}-n^{2}\right)-2 \nu \beta \sigma\left(2 n^{4}-5 n^{3}-5 n^{2}-n\right) \\
& +\nu^{2} \beta^{2}\left(16 n^{4}+36 n^{3}+28 n^{2}+9 n+1\right) .
\end{aligned}
$$

LEMMA 5.1. $a_{n, 0}-a_{n, 1} \beta-a_{n, 2} \beta^{2}>0$ for $n \geqq 11$.

Proof. We shall prove this, dividing into three cases according to the size of $n$.

Case I: $n \geqq 12$. Since we have

$$
\begin{aligned}
& 4 \sigma^{2} \geqq 4 \sigma^{2}(12)=3.127601 \cdots>3.127, \\
& 6 \nu \beta \sigma<\lim _{n \rightarrow \infty} 6 \nu \beta \sigma=12 \sqrt{2}-12=4.970562 \cdots<4.971, \\
& 6 \nu^{2} \beta^{2}<\lim _{n \rightarrow \infty} 6 \nu^{2} \beta^{2}=24(3-2 \sqrt{2})=4.117749 \cdots<4.118,
\end{aligned}
$$


we obtain from (4.26)

$$
\begin{aligned}
a_{n, 0}> & 3.127\left(2 n^{6}-4 n^{5}+5 n^{4}-3 n^{3}\right)-4.971\left(12 n^{5}-8 n^{4}-4 n^{3}+3 n^{2}\right) \\
& -4.118\left(28 n^{4}-16 n^{3}+2 n^{2}+n\right) \\
= & 6.254 n^{6}-72.16 n^{5}-59.901 n^{4}+76.391 n^{3}-23.149 n^{2}-4.118 n
\end{aligned}
$$

for $n \geqq 12$. Since we have

$$
\begin{aligned}
& \sigma^{2}<\lim _{n \rightarrow \infty} \sigma^{2}=1, \\
& 2 \nu \beta \sigma<\lim _{n \rightarrow \infty} 2 \nu \beta \sigma=4 \sqrt{ } 2-4=1.656854 \cdots<1.657, \\
& 3 \nu^{2} \beta^{2}>3\left(\nu^{2} \beta^{2}\right)(12)=1.815702 \cdots>1.815,
\end{aligned}
$$

we obtain from (4.27)

$$
\begin{aligned}
a_{n, 1}< & 8 n^{5}-10 n^{4}+11 n^{3}-9 n^{2}+1.657\left(4 n^{5}-46 n^{4}+23 n^{3}+10 n^{2}-9 n\right) \\
& -1.815\left(32 n^{4}+36 n^{3}-8 n^{2}-3 n+3\right) \\
= & 14.628 n^{5}-144.302 n^{4}-16.229 n^{3}+22.09 n^{2}-9.468 n-5.445
\end{aligned}
$$

for $n \geqq 12$. Since we have

$$
\begin{aligned}
& \sigma^{2} \geqq \sigma^{2}(12)=0.781900 \cdots>0.781, \\
& 2 \nu \beta \sigma>2(\nu \beta \sigma)(12)=1.375838 \cdots>1.375, \\
& \nu^{2} \beta^{2}<\lim _{n \rightarrow \infty} \nu^{2} \beta^{2}=4(3-3 \sqrt{2})=0.686291 \cdots<0.687,
\end{aligned}
$$

we obtain from (5.2)

$$
\begin{aligned}
a_{n, 2}< & -0.781\left(2 n^{4}-n^{3}-n^{2}\right)-1.375\left(2 n^{4}-5 n^{3}-5 n^{2}-n\right) \\
& +0.687\left(16 n^{4}+36 n^{3}+28 n^{2}+9 n+1\right) \\
= & 6.68 n^{4}+32.388 n^{3}+26.892 n^{2}+7.558 n+0.687
\end{aligned}
$$

for $n \geqq 12$. From Lemma 3.5 , we have $\beta<1.415$.

Hence, by (5.3), (5.4), (5.5) and Lemma 4.6 we obtain

$$
\begin{aligned}
& a_{n, 0}-a_{n, 1} \beta-a_{n, 2} \beta^{2}>6.254 n^{6}-72.16 n^{5}-59.901 n^{4}+76.391 n^{3} \\
&-23.149 n^{2}-4.118 n-1.415\left(14.628 n^{5}-144.302 n^{4}-16.229 n^{3}+22.09 n^{2}\right. \\
&-9.468 n-5.445)-2\left(6.68 n^{4}+32.388 n^{3}+26.892 n^{2}+7.558 n+0.687\right) \\
&= 6.254 n^{6}-92.85862 n^{5}+130.92633 n^{4}+34.579035 n^{3}-108.19035 n^{2}
\end{aligned}
$$


48

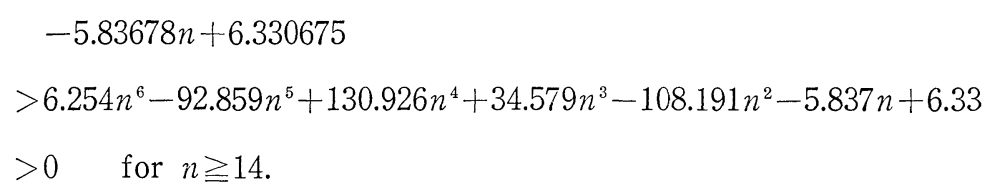

Next, since we have for $12 \leqq n<14$

$$
\begin{aligned}
& 6 \nu \beta \sigma<6(\nu \beta \sigma)(14)=4.243517 \cdots<4.244, \\
& 6 \nu^{2} \beta^{2}<6\left(\nu^{2} \beta^{2}\right)(14)=3.700940 \cdots<3.701, \\
& \sigma^{2}<\sigma^{2}(14)=0.810940 \cdots<0.811, \\
& 2 \nu \beta \sigma<2(\nu \beta \sigma)(14)=1.414505 \cdots<1.415, \\
& \nu^{2} \beta^{2}<\nu^{2} \beta^{2}(14)=0.616823 \cdots<0.617,
\end{aligned}
$$

we obtain

$$
\begin{aligned}
a_{n, 0}> & 3.127\left(2 n^{6}-4 n^{5}+5 n^{4}-3 n^{3}\right)-4.244\left(12 n^{5}-8 n^{4}-4 n^{3}+3 n^{2}\right) \\
& -3.701\left(28 n^{4}-16 n^{3}+2 n^{2}+n\right) \\
= & 6.254 n^{6}-63.436 n^{5}-54.041 n^{4}+66.811 n^{3}-20.134 n^{2}-3.701 n,
\end{aligned}
$$

$$
\begin{aligned}
a_{n, 1}< & 0.811\left(8 n^{5}-10 n^{4}+11 n^{3}-9 n^{2}\right)+1.415\left(4 n^{5}-46 n^{4}+23 n^{3}+10 n^{2}-9 n\right) \\
& -1.815\left(32 n^{4}+36 n^{3}-8 n^{2}-3 n+3\right) \\
= & 12.148 n^{5}-131.28 n^{4}-23.874 n^{3}+21.371 n^{2}-7.29 n-5.445, \\
a_{n, 2}< & -0.781\left(2 n^{4}-n^{3}-n^{2}\right)-1.375\left(2 n^{4}-5 n^{3}-5 n^{2}-n\right) \\
& +0.617\left(16 n^{4}+36 n^{3}+28 n^{2}+9 n+1\right) \\
= & 5.56 n^{4}+29.868 n^{3}+24.932 n^{2}+6.928 n+0.617 .
\end{aligned}
$$

Furthermore, from Lemma 3.5 we have

$$
\beta<\beta(14)=1.392690 \cdots<1.393, \quad \beta^{2}<\beta^{2}(14)=1.939568 \cdots<1.940
$$

for $n<14$. Hence, by $\left(5.3^{\prime}\right),\left(5.4^{\prime}\right),\left(5.5^{\prime}\right)$ and Lemma 4.6 we obtain

$$
\begin{aligned}
& a_{n, 0}-a_{n, 1} \beta-a_{n, 2} \beta^{2} \\
&> 6.254 n^{6}-80.358164 n^{5}+118.04564 n^{4}+42.123562 n^{3}-98.271883 n^{2} \\
&-6.98635 n+6.387905 \\
&> 6.254 n^{6}-80.359 n^{5}+118.045 n^{4}+42.123 n^{3}-98.272 n^{2}-6.987 n+6.387>0
\end{aligned}
$$

for $12 \leqq n<14$. 
Case II : $11 \leqq n<12$. Since we have

$$
\begin{aligned}
& 4 \sigma^{2} \geqq 4 \sigma^{2}(11)=3.055118 \cdots>3.055, \\
& 6 \nu \beta \sigma<6(\nu \beta \sigma)(12)=4.127515 \cdots<4.128 \\
& 6 \nu^{2} \beta^{2}<6\left(\nu^{2} \beta^{2}\right)(12)=3.631405 \cdots<3.632,
\end{aligned}
$$

we obtain from (4.26)

$$
\begin{aligned}
a_{n, 0}> & 3.055\left(2 n^{6}-4 n^{5}+5 n^{4}-3 n^{3}\right)-4.128\left(12 n^{5}-8 n^{4}-4 n^{3}+3 n^{2}\right) \\
& -3.632\left(28 n^{4}-16 n^{3}+2 n^{2}+n\right) \\
= & 6.11 n^{6}-61.756 n^{5}-53.397 n^{4}+65.459 n^{3}-19.648 n^{2}-3.632 n
\end{aligned}
$$

for $11 \leqq n<12$. Since we have

$$
\begin{aligned}
& \sigma^{2}<\sigma^{2}(12)=0.781900 \cdots<0.782, \\
& 2 \nu \beta \sigma<2(\nu \beta \sigma)(12)=1.375838 \cdots<1.376, \\
& 3 \nu^{2} \beta^{2} \geqq 3\left(\nu^{2} \beta^{2}\right)(11)=1.793579 \cdots>1.793,
\end{aligned}
$$

we obtain from (4.27)

$$
\begin{aligned}
a_{n, 1}< & 0.782\left(8 n^{5}-10 n^{4}+11 n^{3}-9 n^{2}\right)+1.376\left(4 n^{5}-46 n^{4}+23 n^{3}+10 n^{2}-9 n\right) \\
& -1.793\left(32 n^{4}+36 n^{3}-8 n^{2}-3 n+3\right) \\
= & 11.76 n^{5}-128.492 n^{4}-24.298 n^{3}+21.066 n^{2}-7.005 n-5.379
\end{aligned}
$$

for $11 \leqq n<12$. Since we have

$$
\begin{aligned}
& \sigma^{2} \geqq \sigma^{2}(11)=0.763779 \cdots>0.763, \\
& 2 \nu \beta \sigma \geqq 2(\nu \beta \sigma)(11)=1.351492 \cdots>1.351, \\
& \nu^{2} \beta^{2}<\nu^{2} \beta^{2}(12)=0.605234 \cdots<0.606
\end{aligned}
$$

for $11 \leqq n<12$, we obtain from (5.2)

$$
\begin{aligned}
a_{n, 2}< & -0.763\left(2 n^{4}-n^{3}-n^{2}\right)-1.351\left(2 n^{4}-5 n^{3}-5 n^{2}-n\right) \\
& +0.606\left(16 n^{4}+36 n^{3}+28 n^{2}+9 n+1\right) \\
= & 5.468 n^{4}+29.334 n^{3}+24.486 n^{2}+6.805 n+0.606
\end{aligned}
$$

for $11 \leqq n<12$.

Now, noticing Lemma 4.6 and Lemma 4.7 , we can prove that the last side of (5.7) is positive for $11.1 \leqq n<12$, by considering the two cases: $11.1 \leqq n<11.2$ and $11.2 \leqq n<12$. Thus, since we have from Lemma 3.5 


$$
\beta<\beta(12)=1.388983 \cdots<1.389, \quad \beta^{2}<\beta^{2}(12)=1.929275 \cdots<1.930
$$

for $n<12$, we obtain

$$
\begin{aligned}
& a_{n, 0}-a_{n, 1} \beta-a_{n, 2} \beta^{2} \\
> & 6.11 n^{6}-78.09064 n^{5}+114.525148 n^{4}+42.594302 n^{3}-96.166654 n^{2} \\
& \quad-7.035705 n+6.301851 \\
> & 6.11 n^{6}-78.091 n^{5}+114.525 n^{4}+42.594 n^{3}-96.167 n^{2}-7.036 n+6.301>0
\end{aligned}
$$

for $11.1 \leqq n<12$.

Finally, we shall prove the above inequality for $11 \leqq n<11.1$. First, we shall show that $a_{n, 1}<0$ in this interval of $n$. Since we have

$$
\begin{aligned}
& \sigma^{2}<\sigma^{2}(11.1)=0.765725 \cdots<0.766, \\
& 2 \nu \beta \sigma<2(\nu \beta \sigma)(11.1)=1.354115 \cdots<1.355, \\
& 3 \nu^{2} \beta^{2} \geqq 3\left(\nu^{2} \beta^{2}\right)(11)=1.793579 \cdots>1.793
\end{aligned}
$$

for $11 \leqq n<11.1$, we obtain from (4.27)

$$
\begin{aligned}
a_{n, 1}< & 0.766\left(8 n^{5}-10 n^{4}+11 n^{3}-9 n^{2}\right)+1.355\left(4 n^{5}-46 n^{4}+23 n^{3}+10 n^{2}-9 n\right) \\
& -1.793\left(32 n^{4}+36 n^{3}-8 n^{2}-3 n+3\right) \\
= & 11.548 n^{5}-127.366 n^{4}-24.957 n^{3}+21 n^{2}-6.816 n-5.379<0
\end{aligned}
$$

for $11 \leqq n<11.1$.

Next, we shall make the evaluations of $a_{n, 0}$ and $a_{n, 2}$ a little more sharper than (5.6) and (5.8). Since we have

$$
\begin{aligned}
& 6 \nu \beta \sigma<6(\nu \beta \sigma)(11.1)=4.062345 \cdots<4.063, \\
& 6 \nu^{2} \beta^{2}<6\left(\nu^{2} \beta^{2}\right)(11.1)=3.591943 \cdots<3.592, \\
& \nu^{2} \beta^{2}<0.598657 \cdots<0.599
\end{aligned}
$$

for $n<11.1$, we obtain from (4.26) and (5.2)

$$
\begin{aligned}
a_{n, 0}>3.055\left(2 n^{6}-4 n^{5}+5 n^{4}-3 n^{3}\right)-4.063\left(12 n^{5}-8 n^{4}-4 n^{3}+3 n^{2}\right) \\
\\
-3.592\left(28 n^{4}-16 n^{3}+2 n^{2}+n\right) \\
=6.11 n^{6}-60.976 n^{5}-52.797 n^{4}+64.559 n^{3}-19.373 n^{2}-3.592 n
\end{aligned}
$$

and

$$
\begin{aligned}
a_{n, 2}< & -0.763\left(2 n^{4}-n^{3}-n^{2}\right)-1.351\left(2 n^{4}-5 n^{3}-5 n^{2}-n\right) \\
& +0.599\left(16 n^{4}+36 n^{3}+28 n^{2}+9 n+1\right)
\end{aligned}
$$


A CERTAIN PROPERTY OF GEODESICS

$$
=5.356 n^{4}+29.082 n^{3}+24.29 n^{2}+6.742 n+0.599
$$

for $11 \leqq n<11.1$. Furthermore, since we have

$$
\beta \geqq \beta(11)=1.386606 \cdots>1.386, \quad \beta^{2}<\beta^{2}(11.1)=1.923393 \cdots<1.924,
$$

we obtain from (5.10), (5.9) and (5.11)

$$
\begin{aligned}
& a_{n, 0}-a_{n, 1} \beta-a_{n, 2} \beta^{2} \\
&> 6.11 n^{6}-76.981528 n^{5}+113.427332 n^{4}+43.195634 n^{3}-95.21296 n^{2} \\
&-7.116632 n+6.302818 \\
&> 6.11 n^{6}-76.982 n^{5}+113.427 n^{4}+43.195 n^{3}-95.213 n^{2}-7.117 n+6.302>0
\end{aligned}
$$

for $11 \leqq n<11.1$.

Thus, we have finished the verification of Case II. $\quad$ Q.E.D. $n=10$.

Remark. By a numerical computation we see that $a_{n, 0}-a_{n, 1} \beta-a_{n, 2} \beta^{2}<0$ at

LEMMA 5.2 . When $n \geqq 11, a_{n, 0}-a_{n, 1} x-a_{n, 2} x^{2}>0$ for $0 \leqq x \leqq \beta$.

Proof. By Lemma 4.2, Lemma 4.4 and Lemma 5.1, the statement of this lemma is evident.

Q.E. D.

Regarding (4.12) we have the following

LEMMA 5.3.

$$
\left.\frac{(n-x)^{n-1} S_{3}(x)}{S_{2}(x)}\right|_{x=1}=B .
$$

Proof. By means of (4.11) and (4.5) (4.8), we have

$$
S_{2}(1)=S_{3}(1)=12(n-1)^{2}\{(n-\beta)+\nu \beta\} .
$$

\section{§6. Properties of $W\left(x, x_{1}\right)$.}

PROPOSITION 1. $W\left(x, x_{1}\right)>0$ for $X^{-1}(\beta) \leqq x<1$ and $X(x) \leqq x_{1}<n$, when $n \geqq 11$.

Proof. By means of Lemma 5.2, we see from (4.20) that the inequality (4.12) is true for $0 \leqq x \leqq \beta$, hence (4.4) is so. Thus, we obtain

$$
(n-x)^{n} F_{2}(x)-\nu \phi(\beta) F_{3}(x)>0
$$

and hence

$$
G^{\prime}(x)>0 \text { for } 0 \leqq x \leqq \beta, x \neq 1
$$

by the argument in $\S 4$. $G(x)$ must be strictly increasing in $0<x<\beta$. By Lemma 
3.5 and Lemma 3.7 we see that the inequality (3.4) must be true for $X^{-1}(\beta) \leqq x<1$ and $X(x)<x_{1}<n$. Accordingly $W\left(x, x_{1}\right)$ is increasing with respect to $x_{1}$ for $X(x)<x_{1}<n$, when $X^{-1}(\beta) \leqq x<1$. From this fact and Lemma 3.2 we obtain

$$
W\left(x, x_{1}\right)>0 \text { for } X^{-1}(\beta) \leqq x<1 \text { and } X(x) \leqq x_{1}<n \text { Q. E. D. }
$$

LEMMA 6.1. The function $W\left(x, x_{1}\right)$ is convex downward with respect to $x_{1}$ for a fixed $x(0<x<1)$, when

and

i) $\max (X(x), 2) \leqq x_{1}<n$;

ii) for $X(x) \leqq x_{1}=Y<2, X^{-1}(2)<x<1$, where

provided $n \geqq 11$.

$$
\left.\frac{\partial}{\partial x_{1}} W\left(x, x_{1}\right)\right|_{x_{1}=Y}=0,
$$

Proof. In the proof of Lemma 3.4, we get

$$
\begin{aligned}
& \frac{1}{\phi(x)} \frac{\partial}{\partial x_{1}} W\left(x, x_{1}\right) \\
& =\left[F_{0}(X)-F_{0}(x)\right] \frac{\left(x_{1}-1\right) n+(n-1) x_{1}}{(n-1) x_{1}{ }^{2}\left(n-x_{1}\right)}-\left[F_{1}(X)-F_{1}(x)\right]\left(x_{1}-1\right)\left(n-x_{1}\right)^{n-2},
\end{aligned}
$$

from which we obtain

$$
\begin{aligned}
\frac{1}{\psi(x)}-\frac{\partial^{2}}{\partial x_{1}{ }^{2}} & W\left(x, x_{1}\right) \\
= & {\left[F_{0}(X)-F_{0}(x)\right] \frac{2\left(n-x_{1}\right)^{2}+(n-1) x_{1}{ }^{3}}{(n-1) x_{1}{ }^{3}\left(n-x_{1}\right)^{2}} } \\
& -\left[F_{1}(X)-F_{1}(x)\right](n-1)\left(2-x_{1}\right)\left(n-x_{1}\right)^{n-3} .
\end{aligned}
$$

Since $F_{0}(X)-F_{0}(x)$ and $F_{1}(X)-F_{1}(x)$ are positive for $0<x<1$, we obtain easily that

$$
\frac{\partial^{2} W\left(x, x_{1}\right)}{\partial x_{1}^{2}}>0 \text { for } 2 \leqq x_{1}<n \text {. }
$$

Next, regarding the second statement, we see immediately from (6.1) that $\frac{\partial}{\partial x_{1}} W\left(x, x_{1}\right)$ vanishes if and only if

$$
\frac{F_{0}(X)-F_{0}(x)}{F_{1}(X)-F_{1}(x)}=\frac{(n-1) x_{1}{ }^{2}\left(n-x_{1}\right)^{n-1}}{n+(n-1) x_{1}}\left(:=\eta\left(x_{1}\right)\right) .
$$

From (6.2) we see that $\partial^{2} W\left(x, x_{1}\right) / \partial x_{1}{ }^{2}>0$ if and only if

$$
\frac{F_{0}(X)-F_{0}(x)}{F_{1}(X)-F_{1}(x)}>\frac{(n-1)^{2} x_{1}{ }^{3}\left(2-x_{1}\right)\left(n-x_{1}\right)^{n-1}}{2\left(n-x_{1}\right)^{2}+(n-1) x_{1}{ }^{3}} \text {. }
$$


Now, we factorize the right hand side of (6.4) as

$$
\frac{(n-1) x_{1}\left(2-x_{1}\right)\left\{n+(n-1) x_{1}\right\}}{2\left(n-x_{1}\right)^{2}+(n-1) x_{1}{ }^{3}} \cdot \frac{(n-1) x_{1}{ }^{2}\left(n-x_{1}\right)^{n-1}}{n+(n-1) x_{1}},
$$

the first factor of which is decreasing for $\frac{\sqrt{3}}{\sqrt{2}}<x_{1}$ and takes the value 1 at $x_{1}=\beta$. In fact, we can easily show that $x_{1}\left(2-x_{1}\right)\left\{n+(n-1) x_{1}\right\}$ is decreasing and $2\left(n-x_{1}\right)^{2}+(n-1) x_{1}{ }^{3}$ is increasing for $\frac{\sqrt{3}}{\sqrt{2}}<x_{1}$. Next, using the equality

$$
(n-1) \beta^{2}=2(n-\beta)
$$

by Lemma 3.5 , we have

$$
\begin{aligned}
& \frac{(n-1) \beta(2-\beta)\{n+(n-1) \beta\}}{2(n-\beta)^{2}+(n-1) \beta^{3}}=\frac{(n-1)(2-\beta)\{n \beta+2(n-\beta)\}}{2(n-\beta)^{2}+2 \beta(n-\beta)} \\
= & \frac{(n-1)\left\{4 n-4 \beta-(n-2) \beta^{2}\right\}}{2 n(n-\beta)}=1 .
\end{aligned}
$$

Going back to the proof of ii), we assume that (6.3) holds at $x_{1}=Y, X(x) \leqq$ $Y<n, 0<x<1$. When $Y \geqq 2, W\left(x, x_{1}\right)$ is convex downward at $x_{1}=Y$ by i). When $Y<2$, it must be $X^{-1}(2)<x<1$.

On the other hand, from Lemma 3.6 we have

$$
\beta(n)>\sqrt{\frac{2 n}{n+1}} \text { for } n>1
$$

and

$$
\sqrt{\frac{2 n}{n+1}}>\frac{\sqrt{3}}{\sqrt{2}} \text { for } n \geqq 3
$$

Now, assuming $n \geqq 11$, we see that it must be $X^{-1}(2)<x<X^{-1}(\beta)$ by Proposition 1. Hence we have

$$
\frac{\sqrt{3}}{\sqrt{2}}<\beta \quad \text { and } \quad \beta<X(x) \leqq Y<2
$$

and so

$$
0<\frac{(n-1) Y(2-Y)\{n+(n-1) Y\}}{2(n-Y)^{2}+(n-1) Y^{3}}<1 .
$$

Therefore we obtain

$$
\frac{F_{0}(X)-F_{0}(x)}{F_{0}(X)-F_{0}(x)}>\frac{(n-1)^{2} Y^{3}(2-Y)(n-Y)^{n-1}}{2(n-Y)^{2}+(n-1) Y^{3}},
$$

which shows that $W\left(x, x_{1}\right)$ is convex downward with respect to $x_{1}$ at $x_{1}=Y$.

Q.E.D. 
This lemma and Lemma 3.2 imply immediately the following

LEMMA 6.2. When $n \geqq 11$, for a fixed $x(0<x<1)$ the function $W\left(x, x_{1}\right)$ takes its critical value with respect $x_{1}$ at most one point in the interval $X(x) \leqq x_{1}<n$, which becomes the minimum value.

LEMMA 6.3. For a sufficiently small fixed $x(0<x<1), W(X, x)$ as function of $x_{1}$ takes its critical value in the interval $X(x)<x_{1}<n$.

Proof. By means of Lemma 3.4 or Lemma 6.1, we see that $W\left(x, x_{1}\right)$ is critical with respect to $x_{1}$ at $x_{1}=Y$ if and only if

$$
\begin{gathered}
F_{0}(X)-F_{0}(x)=\frac{(n-1) Y^{2}(n-Y)^{n-1}}{n+(n-1) Y}\left\{F_{1}(X)-F_{1}(x)\right\}, \\
X(x) \leqq Y<n .
\end{gathered}
$$

For $0<x<X^{-1}(\beta)$, we have $\beta<X(x)$ and then from the fact described in the proof of Lemma 3.5, we see that there exists $Y$ satisfying (6.6) if and only if

$$
F_{0}(X)-F_{0}(x) \leqq \frac{(n-1) X^{2}(n-X)^{n-1}}{n+(n-1) X}\left\{F_{1}(X)-F_{1}(x)\right\},
$$

which is equivalent to

$$
\begin{aligned}
& \frac{X \sqrt{n-X} f_{0}(X)}{(X-1)^{3}}-\frac{x \sqrt{n-x} f_{0}(x)}{(x-1)^{3}} \\
& \leqq \frac{(n-1) X}{n+(n-1) X}\left\{\frac{X^{2} f_{1}(X)}{(1-X)^{3} \sqrt{n-X}}-\frac{x^{2} f_{1}(x)}{(1-x)^{3} \sqrt{n-x}}\right\}
\end{aligned}
$$

by (3.2) and (3.3).

Now, we obtain easily from (2.10)

$$
\lim _{x \rightarrow 0}\left\{\frac{X \sqrt{n-X} f_{0}(X)}{(X-1)^{3}}-\frac{x \sqrt{n-x} f_{0}(x)}{(x-1)^{3}}\right\}=0
$$

and

$$
\begin{aligned}
& \lim _{x \rightarrow 0} \frac{(n-1) X}{n+(n-1) X}\left\{\frac{X^{2} f_{1}(X)}{(1-X)^{3} \sqrt{n-X}}-\frac{x^{2} f_{1}(x)}{(1-x)^{3} \sqrt{n-x}}\right\}=-\frac{n(2 n-1) B}{n-1} \lim _{x \rightarrow 0} \frac{1}{\sqrt{n-X}} \\
& =+\infty .
\end{aligned}
$$

Hence, (6.7) must hold for sufficiently small $x>0$ without the equality sign.

Q.E. D.

Taking these lemmas into consideration, for each $x(0<x<1)$ let $Y=Y(x)$ be the value of $x_{1}$ where $W\left(x, x_{1}\right)$ takes its minimum in the interval $X(x) \leqq x_{1}<n$.

LEMMA 6.4. We have 


$$
\lim _{x \rightarrow+0} W(x, X(x))=\infty \quad(n>2) .
$$

Proof. From $x(n-x)^{n-1}=X(n-X)^{n-1}$, we have

$$
x=\frac{1}{n^{n-2}}(n-X)^{n-1} \text { near } x=0,
$$

from which we obtain

$$
\lim _{x \rightarrow+0} \frac{x}{n-X}=0
$$

We have also from (1.4)

$$
\lim _{x \rightarrow+0}(n-X) \lambda(X)=\lim _{x \rightarrow n}\{(n-X) \log (n-X)+n-1\}=n-1 .
$$

Now, since we have

$$
\begin{aligned}
W(x, X(x))= & \frac{x \sqrt{n-x} f_{0}(x)}{(x-1)^{3}}\{\lambda(X)-\lambda(x)\} \\
& +\left[\frac{X \sqrt{n-X} f_{0}(X)}{(X-1)^{3}}-\frac{x \sqrt{n-x} f_{0}(x)}{(x-1)^{3}}\right] \frac{n(X-1)^{2}}{(n-1) X(n-X)} \\
= & \frac{\sqrt{n-x} f_{0}(x)}{(x-1)^{3}}\left\{(n-X) \lambda(X) \frac{x}{n-X}-x \lambda(x)\right\} \\
& +\frac{n f_{0}(X)}{(n-1)(X-1)} \cdot \frac{1}{\sqrt{n-X}}-\frac{n \sqrt{n-x} f_{0}(x)(X-1)^{2}}{(n-1)(x-1)^{3} X} \cdot \frac{x}{n-X},
\end{aligned}
$$

we obtain

$$
\begin{aligned}
\lim _{x \rightarrow+0} W(x, X(x))= & -\sqrt{n} f_{0}(0)\left[(n-1) \lim _{x \rightarrow+0} \frac{x}{n-X}-0\right] \\
& +\frac{n f_{0}(n)}{(n-1)^{2}} \lim _{x \rightarrow+0} \frac{1}{\sqrt{n-X}}+(n-1) \sqrt{n} f_{0}(0) \lim _{x \rightarrow+0} \frac{x}{n-X} \\
= & \frac{n f_{0}(n)}{(n-1)^{2}} \lim _{x \rightarrow+0} \frac{1}{\sqrt{n-X}}=+\infty
\end{aligned}
$$

LEMMA 6.5. We have

$$
\lim _{x \rightarrow+0} W(x, Y(x))=-\infty .
$$

Proof. By Lemma 6.3 we have (6.6) at $Y=Y(x)$ for sufficiently small $x>0$. Hence, we have

$$
\begin{aligned}
\frac{\phi(Y)}{\phi(X)} & =\frac{n+(n-1) Y}{(n-1) Y} \cdot \frac{F_{0}(X)-F_{0}(x)}{\left\{F_{1}(X)-F_{1}(x)\right\} \psi(x)} \\
& =\frac{n+(n-1) Y}{(n-1) Y} \cdot \frac{\frac{f_{0}(X)}{(n-X)^{n-3 / 2}(X-1)^{3}}-\frac{f_{0}(x)}{(n-x)^{n-3 / 2}(x-1)^{3}}}{\left\{\frac{f_{1}(X)}{(n-X)^{2 n-3 / 2}(1-X)^{3}}-\frac{f_{1}(x)}{(n-x)^{2 n-3 / 2}(1-x)^{3}}\right\} \psi(x)}
\end{aligned}
$$




$$
=\frac{n+(n-1) Y}{(n-1) Y} \cdot \frac{\frac{f_{0}(X)}{(X-1)^{3}}-\frac{f_{0}(x)}{(x-1)^{3}}\left(\frac{n-X}{n-x}\right)^{n-3 / 2}}{\frac{X f_{1}(X)}{(1-X)^{3}}-\frac{x f_{1}(X)}{(1-x)^{3}}\left(\frac{n-X}{n-x}\right)^{n-1 / 2}} \cdot(n-X),
$$

from which we obtain near $x=0$

$$
\frac{\phi(Y)}{\phi(X)}=\frac{n^{2}}{(n-1) n} \cdot \frac{f_{0}(n)}{-n f_{1}(n)} \cdot(n-X)=\frac{-f_{0}(n)}{(n-1) f_{1}(n)}(n-X) .
$$

Since we have from $(2.10)$

we obtain

$$
f_{0}(n)=(n-1) B, \quad f_{1}(n)=-(n-1)(2 n-1) B,
$$

$$
\frac{\phi(Y)}{\psi(X)}=\frac{Y(n-Y)^{n-1}}{X(n-X)^{n-1}}=\frac{1}{(n-1)(2 n-1)}(n-X)
$$

near $x=0$, which implies

$$
\lim _{x \rightarrow+0} \frac{\phi(Y)}{\phi(X)}=+0 .
$$

Now, we have from (3.1)

$$
\begin{aligned}
W(x, & Y(x))=\frac{x \sqrt{n-x} f_{0}(x)}{(x-1)^{3}}\{\lambda(X)-\lambda(x)\} \\
+ & {\left[\frac{X \sqrt{n-X} f_{0}(X)}{(X-1)^{3}}-\frac{x \sqrt{n-x} f_{0}(x)}{(x-1)^{3}}\right]\left\{\frac{n}{n-1}-\frac{(Y-1)^{2}}{Y(n-Y)}-\lambda(Y)+\lambda(X)\right\} } \\
& -\frac{1}{n}\left[\frac{X^{2} f_{1}(X)}{(1-X)^{3} \sqrt{n-X}}-\frac{x^{2} f_{1}(x)}{(1-x)^{3} \sqrt{n-x}}\right]\left\{1-\frac{\phi(Y)}{\phi(X)}\right\} \\
= & \frac{\sqrt{n-x} f_{0}(X)}{(x-1)^{3}}(n-X) \lambda(X) \frac{x}{n-X}-\frac{x \sqrt{n-x} f_{0}(x) \lambda(x)}{(x-1)^{3}} \\
+ & {\left[(n-X)\left\{\frac{n}{n-1} \frac{(Y-1)^{2}}{Y(n-Y)}-\lambda(Y)\right\}+(n-X) \lambda(X)\right] } \\
& \cdot\left[\frac{X f_{0}(X)}{(X-1)^{3}} \cdot \frac{1}{\sqrt{n-X}}-\frac{\sqrt{n-x} f_{0}(x)}{(x-1)^{3}} \cdot \frac{x}{n-X}\right] \\
- & \frac{1}{n}\left\{1-\frac{\phi(Y)}{\phi(X)}\right\} \frac{X^{2} f_{1}(X)}{(1-X)^{3}} \cdot \frac{1}{\sqrt{n-X}}+\frac{1}{n}\left\{1-\frac{\phi(Y)}{\psi(X)}\right\} \frac{x^{2} f_{1}(x)}{(1-x)^{3} \sqrt{n-x}} .
\end{aligned}
$$

On the other hand, near $x=0$ we obtain from (6.10)

$$
n-Y=\left\{\frac{1}{(n-1)(2 n-1)}\right\}^{1 /(n-1)}(n-X)^{n /(n-1)}
$$

or 


$$
n-X=\{(n-1)(2 n-1)\}^{1 / n}(n-Y)^{(n-1) / n}
$$

and

$$
\begin{aligned}
& \frac{n}{n-1} \frac{(Y-1)^{2}}{Y}-(n-Y) \lambda(Y) \\
= & \frac{1}{n-1} \frac{(n-1)^{2}-2(n-1)(n-Y)+(n-Y)^{2}}{1-\frac{n-Y}{n}}-(n-Y) \log (n-Y)-(n-1) \\
= & (n-1)\left\{1-\frac{2(n-Y)}{n-1}+\frac{(n-Y)^{2}}{(n-1)^{2}}\right\}\left\{1+\frac{n-Y}{n}+\left(\frac{n-Y}{n}\right)^{2}+\cdots\right\} \\
& -(n-Y) \log (n-Y)-(n-1) \\
= & (n-Y)\left[-\log (n-Y)-\frac{n+1}{n}+\frac{1}{n(n-1)}\left\{\frac{n-Y}{n}+\left(\frac{n-Y}{n}\right)^{2}+\cdots\right\} .\right.
\end{aligned}
$$

Hence we have

$$
\begin{aligned}
& \lim _{x \rightarrow+0}(n-X)\left\{\frac{n}{n-1} \cdot \frac{(Y-1)^{2}}{Y(n-Y)}-\lambda(Y)\right\} \\
= & \lim _{x \rightarrow+0} \frac{n-X}{n-Y}\left\{\frac{n}{n-1} \frac{(Y-1)^{2}}{Y}-(n-Y) \lambda(Y)\right\} \\
= & \lim _{x \rightarrow+0}(n-X)\left[-\log (n-Y)-\frac{n+1}{n}+\frac{1}{n(n-1)}\left\{\frac{n-Y}{n}+\left(\frac{n-Y}{n}\right)^{2}+\cdots\right\}\right. \\
= & \lim _{x \rightarrow+0}-(n-X) \log (n-Y)=-\{(n-1)(2 n-1)\}^{1 / n} \lim _{x \rightarrow+0}(n-Y) \log (n-Y) \\
= & 0
\end{aligned}
$$

i. e.

$$
\lim _{x \rightarrow+0}(n-X)\left\{\frac{n}{n-1} \frac{(Y-1)^{2}}{Y(n-Y)}-\lambda(Y)\right\}=0 .
$$

Using these facts, we obtain from the expression above of $W(x, Y(x))$

$$
\begin{aligned}
& \lim _{x \rightarrow+0} W(x, Y(x))=\frac{\sqrt{n} f_{0}(0)}{-1}(n-1) \lim _{x \rightarrow+0} \frac{x}{n-X}-0 \\
& +\lim _{x \rightarrow+0}\left\{\left[(n-X)\left\{\frac{n}{n-1} \frac{(Y-1)^{2}}{Y(n-Y)}-\lambda(Y)\right\}+(n-X) \lambda(X)\right] \frac{X f_{0}(X)}{(X-1)^{3} \sqrt{n-X}}\right. \\
& \left.\quad-\frac{1}{n}\left\{1-\frac{\psi(Y)}{\psi(X)}\right\} \frac{X^{2} f_{1}(X)}{(1-X)^{3}}\right\} \cdot \frac{1}{\sqrt{n-X}}+0 \\
& =\lim _{x \rightarrow+0}\left\{(n-1) \frac{n f_{0}(n)}{(n-1)^{3}}-\frac{1}{n} \frac{n^{2} f_{1}(n)}{(1-n)^{3}}\right\} \frac{1}{\sqrt{n-X}}
\end{aligned}
$$




$$
=\frac{n}{(n-1)^{3}}\left\{(n-1) f_{0}(n)+f_{1}(n)\right\} \lim _{x \rightarrow+0} \frac{1}{\sqrt{n-X}} .
$$

Since we have

$$
(n-1) f_{0}(n)+f_{1}(n)=\left\{(n-1)^{2}-(n-1)(2 n-1)\right\} B=-n(n-1) B<0,
$$

we obtain finally

$$
\lim _{x \rightarrow+0} W(x, Y(x))=-\infty \quad \text { Q.E. D. }
$$

\section{$\S 7$. A device in the main integral.}

By virtue of the properties of $W\left(x, x_{1}\right)$, especially described in Lemma 6.4 and Lemma 6.5, which are not desirable for our way to prove Conjecture $C$ by making use of Lemma 3.1, we shall try to find a more convenient form of the main integral.

First of all, we show that $W\left(x, x_{1}\right)$ given by (3.1) can be written as

$$
\begin{aligned}
W\left(x, x_{1}\right)= & \phi(x) F_{0}(x)\{\lambda(X)-\lambda(x)\} \\
& +\psi(x)\left[F_{0}(X)-F_{0}(x)\right] \cdot\left\{\frac{n}{n-1} \frac{\left(x_{1}-1\right)^{2}}{x_{1}\left(n-x_{1}\right)}-\lambda\left(x_{1}\right)+\lambda(X)\right\} \\
& -\frac{\phi(x)}{n}\left[F_{1}(X)-F_{1}(x)\right] \cdot\left\{\psi(x)-\psi\left(x_{1}\right)\right\} \\
= & \psi(X)\left[F_{0}(X)\left\{\lambda(X)-\tilde{\lambda}\left(x_{1}\right)\right\}-\frac{1}{n} F_{1}(X)\left\{\psi(X)-\psi\left(x_{1}\right)\right\}\right] \\
& -\psi(x)\left[F_{0}(x)\left\{\lambda(x)-\tilde{\lambda}\left(x_{1}\right)\right\}-\frac{1}{n} F_{1}(x)\left\{\psi(x)-\psi\left(x_{1}\right)\right\}\right],
\end{aligned}
$$

where we set

$$
\tilde{\lambda}(x):=\lambda(x)-\frac{n}{n-1} \cdot \frac{(x-1)^{2}}{x(n-x)}=\log (n-x)+\frac{n x-1}{(n-1) x} .
$$

Here we introduce a complex valued function by

$$
E\left(z, x_{1}\right):=F_{0}(z)\left\{\lambda(z)-\tilde{\lambda}\left(x_{1}\right)\right\}-\frac{1}{n} F_{1}(z)\left\{\psi(z)-\phi\left(x_{1}\right)\right\},
$$

which is analytic with respect to $z$ with singularity only at $z=n$. Then, we have

$$
W\left(x, x_{1}\right)=\psi(x)\left\{E\left(X, x_{1}\right)-E\left(x, x_{1}\right)\right\}
$$

and going back to $\S 3$ we obtain easily the formula: 


$$
\begin{aligned}
\frac{\partial \Omega(\tau, n)}{\partial n} & =\frac{\sqrt{c}}{2 b^{2} \sqrt{n}} \int_{x_{0}}^{x_{1}} \frac{(1-x)(n-x)^{n-2} E\left(x, x_{1}\right) d x}{\sqrt{x(n-x)^{n-1}-c}} \\
& =-\frac{\sqrt{c}}{4 b^{2} \sqrt{ } n} \int_{r} \frac{(1-z)(n-z)^{n-2} E\left(z, x_{1}\right) d z}{\sqrt{z(n-z)^{n-1}-c}} .
\end{aligned}
$$

Proposition 2. We have

$$
\int_{\gamma} \frac{(1-z)(n-z)^{n-2} E\left(z, x_{1}\right) d z}{\sqrt{z(n-z)^{n-1}-c}}=\frac{2}{n} \int_{x_{0}}^{x_{1}} \frac{\sqrt{x(n-x)^{n-1}-c} N\left(x, x_{1}\right) d x}{(x-1)^{4}(n-x)^{n+1 / 2}}
$$

where

$$
N\left(x, x_{1}\right)=(n-x) F_{2}(x)\left\{\lambda(x)-\tilde{\lambda}\left(x_{1}\right)\right\}+3(x-1)^{2} f_{0}(x)-2 n(x-1)^{3}\{B-\psi(x)\} .
$$

Proof. Noticing $c=\phi\left(x_{1}\right)$ on the Riemann surface $\mathscr{I}$ and setting $c^{*}=\tilde{\lambda}\left(x_{1}\right)$, we obtain

$$
\begin{aligned}
& \frac{(1-z)(n-z)^{n-2} E\left(z, x_{1}\right) d z}{\sqrt{\phi(z)-c}} \\
= & \frac{(1-z)(n-z)^{n-2}}{w}\left[F_{0}(z)\left\{\lambda(z)-c^{*}\right\}-\frac{1}{n} F_{1}(z)\{\psi(z)-c\}\right] d z \\
= & \frac{2}{n} F_{0}(z)\left\{\lambda(z)-c^{*}\right\} d w-\frac{1}{n}(1-z)(n-z)^{n-2} F_{1}(z) w d z \\
= & \frac{2}{n} d\left[F_{0}(z)\left\{\lambda(z)-c^{*}\right\} w\right] \\
& -\frac{w}{n}\left[2 d\left(F_{0}(z)\left\{\lambda(z)-c^{*}\right\}\right)+(1-z)(n-z)^{n-2} F_{1}(z) d z\right] .
\end{aligned}
$$

Since we have from (3.2), (3.3) and (1.5), (2.7) in [12]

$$
\frac{d}{d z}\left(F_{0}(z)\left\{\lambda(z)-c^{*}\right\}\right)=\frac{F_{2}(z)\left\{\lambda(z)-c^{*}\right\}}{2(z-1)^{4}(n-z)^{n-1 / 2}}+F_{0}(z) \frac{z-1}{(n-z)^{2}}
$$

and

$$
\begin{aligned}
& 2 d\left(F_{0}(z)\left\{\lambda(z)-c^{*}\right\}\right)+(1-z)(n-z)^{n-2} F_{1}(z) d z \\
& =\left[\frac{F_{2}(z)\left\{\lambda(z)-c^{*}\right\}}{(z-1)^{4}(n-z)^{n-1 / 2}}+\frac{2 f_{0}(z)}{(z-1)^{2}(n-z)^{n+1 / 2}}+\frac{f_{1}(z)}{(1-z)^{2}(n-z)^{n+1 / 2}}\right] d z \\
& =\frac{1}{(z-1)^{4}(n-z)^{n+1 / 2}}\left[(n-z) F_{2}(z)\left\{\lambda(z)-c^{*}\right\}+(z-1)^{2}\left\{2 f_{0}(z)+f_{1}(z)\right\}\right] d z,
\end{aligned}
$$

we obtain the formula

$$
\int_{r} \frac{(1-z)(n-z)^{n-2} E\left(z, x_{1}\right) d z}{\sqrt{z(n-z)^{n-1}-c}}
$$




$$
=-\frac{1}{n} \int_{r} \frac{\sqrt{z(n-z)^{n-1}-c}}{(z-1)^{4}(n-z)^{n+1 / 2}}\left[(n-z) F_{2}(z)\left\{\lambda(z)-c^{*}\right\}+(z-1)^{2}\left\{2 f_{0}(z)+f_{1}(z)\right\}\right] d z .
$$

On the other hand, we have from (2.10)

$$
f_{1}(z)=f_{0}(z)-2 n(z-1)\{B-\psi(z)\},
$$

and so

$$
2 f_{0}(z)+f_{1}(z)=3 f_{0}(z)-2 n(z-1)\{B-\phi(z)\} .
$$

Hence setting

$$
\begin{aligned}
N\left(z, x_{1}\right):= & (n-z) F_{2}(z)\left\{\lambda(z)-\tilde{\lambda}\left(x_{1}\right)\right\}+3(z-1)^{2} \int_{0}(z) \\
& -2 n(z-1)^{3}\{B-\phi(z)\},
\end{aligned}
$$

(7.5) becomes

$$
\begin{aligned}
& \int_{r} \frac{(1-z)(n-z)^{n-2} E\left(z, x_{1}\right) d z}{\sqrt{z(n-z)^{n-1}-c}} \\
& =-\frac{1}{n} \int_{r} \frac{\sqrt{z(n-z)^{n-1}-c} N\left(z, x_{1}\right) d z}{(z-1)^{4}(n-z)^{n+1 / 2}}=\frac{2}{n} \int_{x_{0}}^{x_{1}} \frac{\sqrt{x(n-x)^{n-1}-c} N\left(x, x_{1}\right) d x}{(x-1)^{4}(n-x)^{n+1 / 2}}
\end{aligned}
$$

Remark. By means of the remark at the end of $\S 2$ in $[12], F_{2}(x)$ has a zero point of order at least 4 at $x=1$. And $f_{0}(x)$ has a zero point of order 3 by Lemma 2.2 and $B-\phi(x)$ has a zero point of order 2 at $x=1$.

LEMMA 7.1. $\tilde{\lambda}(x)(0<x<n)$ has the following propertıes:

(i ) $\lim _{x \rightarrow+0} \tilde{\lambda}(x)=\lim _{x \rightarrow n-0} \tilde{\lambda}(x)=-\infty$,

(ii) $\frac{d \tilde{\lambda}(x)}{d x}=-\frac{(x-1)\{n+(n-1) x\}}{(n-1) x^{2}(n-x)}$,

(iii) $\frac{d^{2} \tilde{\lambda}(x)}{d x^{2}}=-\frac{2}{(n-1) x^{3}}-\frac{1}{(n-x)^{2}}<0$.

Proof. We obtain this lemma immediately from (7.1).

LEMMA 7.2. $N\left(x, x_{1}\right)\left(0<x<n, 1 \leqq x_{1}<n\right)$ has the following properties:

(i) $\lim _{x \rightarrow n-0} N\left(x, x_{1}\right)=+\infty$ for $x \neq 1$,

(ii) $N\left(1, x_{1}\right)=0$ for $1 \leqq x_{1}<n$,

(iii) $\lim _{x \rightarrow+0} N(x, X(x))=+\infty$, 
(iv) $\lim _{x \rightarrow n-0} N(x, x)=0$,

(v) $N(x, x)<0$ for $x>1$ sufficiently near $x=1$,

(vi) $\frac{\partial N\left(x, x_{1}\right)}{\partial x_{1}}>0$ for $x \neq 1,1<x_{1}<n$.

Proof. (i) is derived easily from Lemma 7.1 and the fact $F_{2}(x)>0$ for $0 \leqq x<n, x \neq 1$ (Proposition 1 in [12]). (ii) is evident.

Next, we shall prove (iii) and (iv). We have easily

$$
\lim _{x \rightarrow+0} N(x, X(x))=n F_{2}(0) \lim _{x \rightarrow 0}(-\lambda(X(x)))+n F_{2}(0) \lambda(0)+3 f_{0}(0)+2 n B=+\infty,
$$

and by $(7.1)$ and $F_{2}(n)=(n-1)^{2}(2 n-3) B$ we have

$$
\begin{aligned}
& \lim _{x \rightarrow n-0} N(x, x)=\lim _{x \rightarrow n-0} F_{2}(x) \frac{n(x-1)^{2}}{(n-1) x}+3(n-1)^{2} f_{0}(n)-2 n(n-1)^{3} B \\
& =(n-1) F_{2}(n)+3(n-1)^{3} B-2 n(n-1)^{3} B=0 .
\end{aligned}
$$

From Lemma 2.3 in [12] and Lemma 4.1 in [11] we obtain

$$
\lim _{x \rightarrow 1} \frac{f_{0}(x)}{(x-1)^{2}}=\frac{1}{6} n(2 n-1)(n-1)^{n-2}, \quad \lim _{x \rightarrow 1} \frac{B-\psi(x)}{(x-1)^{2}}=\frac{n(n-1)^{n-2}}{2} .
$$

Since we have

$$
N(x, x)=\frac{n}{n-1} \frac{(x-1)^{2}}{x} F_{2}(x)+3(x-1)^{2} f_{0}(x)-2 n(x-1)^{3}\{B-\psi(x)\},
$$

we obtain

$$
\begin{aligned}
\lim _{x \rightarrow 1+0} \frac{N(x, x)}{(x-1)^{5}}= & \frac{n}{n-1} \lim _{x \rightarrow 1+0} \frac{F_{2}(x)}{(x-1)^{3}}+\frac{1}{2} n(2 n-1)(n-1)^{n-2} \\
& -n^{2}(n-1)^{n-2}=0-\frac{1}{2} n(n-1)^{n-2}<0,
\end{aligned}
$$

because $F_{2}(x)$ has a zero point of order at least 4 . This fact implies immediately (v).

Finally, from Lemma 7.1 we obtain

$$
\begin{aligned}
& \frac{\partial N\left(x, x_{1}\right)}{\partial x_{1}}=(n-x) F_{2}(x) \frac{\left(x_{1}-1\right)\left\{n+(n-1) x_{1}\right\}}{(n-1) x_{1}^{2}\left(n-x_{1}\right)}>0 \\
& \quad \text { for } 0<x<n, x \neq 1 \text { and } 1<x_{1}<n . \quad \text { Q. E. D. }
\end{aligned}
$$

Taking note of the facts in Lemma 7.2, we shall provide for the following

Proposition 3. We have 


$$
\int_{\gamma} \frac{(1-z)(n-z)^{n-2} E\left(z, x_{1}\right) d z}{\sqrt{z(n-z)^{n-1}-c}}=\frac{2}{n} \int_{x_{0}}^{1} \frac{(1-x) \sqrt{x(n-x)^{n-1}-c}}{x^{2}(n-x)^{n}} V\left(x, x_{1}\right) d x,
$$

where

$$
V\left(x, x_{1}\right):=\frac{x^{2} N\left(x, x_{1}\right)}{(1-x)^{5} \sqrt{n-x}}+\frac{X^{2} N\left(X(x), x_{1}\right)}{(X-1)^{5} \sqrt{n-X}} \text { for } 0<x<1
$$

Proof. In fact we have

$$
\begin{aligned}
\int_{x_{0}}^{x_{1}} \frac{\sqrt{\psi(x)-c} N\left(x, x_{1}\right) d x}{(x-1)^{4}(n-x)^{n+1 / 2}}= & \int_{x_{0}}^{1} \frac{x \sqrt{\psi(x)-c} N\left(x, x_{1}\right)}{(1-x)^{5}(n-x)^{n-1 / 2}} \cdot \frac{1-x}{x(n-x)} d x \\
& +\int_{1}^{x_{1}} \frac{X \sqrt{\psi(X)-c} N\left(X, x_{1}\right)}{(X-1)^{5}(n-X)^{n-1 / 2}} \cdot \frac{X-1}{X(n-X)} d X .
\end{aligned}
$$

By means of (2.12) and (2.13), the second integral of the right hand side of the above equality becomes

$$
\begin{aligned}
& \int_{x_{0}}^{1} \frac{X \sqrt{\phi(x)-c} N\left(X, x_{1}\right)}{(X-1)^{5}(n-X)^{n-1 / 2}} \cdot \frac{1-x}{x(n-x)} d x \\
& =\int_{x_{0}}^{1} \frac{\sqrt{\psi(x)-c} X^{2} N\left(X, x_{1}\right)}{\psi(x)(X-1)^{5} \sqrt{n-X}} \cdot \frac{1-x}{x(n-x)} d x .
\end{aligned}
$$

Hence, we obtain

$$
\begin{aligned}
& \int_{x_{0}}^{x_{1}} \frac{\sqrt{\psi(x)-c} N\left(x, x_{1}\right) d x}{(x-1)^{4}(n-x)^{n+1 / 2}}=\int_{x_{0}}^{1} \frac{(1-x) \sqrt{\psi(x)-c}}{x^{2}(n-x)^{n}} \\
& \quad \cdot\left[\frac{x^{2} N\left(x, x_{1}\right)}{(1-x)^{5} \sqrt{n-x}}+\frac{X^{2} N\left(X, x_{1}\right)}{(X-1)^{5} \sqrt{n-X}}\right] d x=\int_{x_{0}}^{1} \frac{(1-x) \sqrt{\psi(x)-c} V\left(x, x_{1}\right) d x}{x^{2}(n-x)^{n}},
\end{aligned}
$$

which implies this proposition.

Q.E. D.

Formula (7.4), Proposition 2, Proposition 3 and Lemma 7.2 imply $\frac{\partial \Omega(\tau, n)}{\partial n}<0$, if we can prove that $V(x, X(x)) \geqq 0$ for $0<x<1$.

\section{$\S 8$. Properties of $V\left(x, x_{1}\right)$.}

LEMMA 8.1. $V\left(x, x_{1}\right)\left(0<x<1,1<x_{1}<n\right)$ has the following properties:

(i) $\lim _{x_{1} \rightarrow n-0} V\left(x, x_{1}\right)=+\infty$ for $0<x<1$;

(ii) $V\left(x, x_{1}\right)$ is increasing with respect to $x_{1}$ for each $x(0<x<1)$;

(iii) When $n>2, \lim _{x \rightarrow+0} V(x, X(x))=0$ and furthermore when $n>\frac{5+\sqrt{13}}{4}=2.15$, $V(x, X(x))>0$ near $x=0$;

(iv) When $n>2, \lim _{x \rightarrow 1-0} V(x, X(x))=0$ and furthermore when $n>\frac{1+\sqrt{13}}{2}=2.30$, $V(x, X(x))>0$ near $x=1$. 
Proof. From (7.10) and Lemma 7.2 we obtain easily (i) and (ii). have

Now, we shall estimate $V(x, X(x))$ near $x=0$. From $(7.7),(7.9)$ and (7.10) we

$$
\begin{aligned}
V(x, X(x))= & \frac{x^{2} N(x, X)}{(1-x)^{5} \sqrt{n-x}}+\frac{X^{2} N(X, X)}{(X-1)^{5} \sqrt{n-X}} \\
= & \frac{x^{2} \sqrt{n-x}}{(1-x)^{5}} F_{2}(x)\{\lambda(x)-\tilde{\lambda}(X)\}-\frac{3 x^{2} f_{0}(x)}{(x-1)^{3} \sqrt{n-x}}+\frac{2 n x^{2}\{B-\psi(x)\}}{(1-x)^{2} \sqrt{n-x}} \\
& +\frac{n}{n-1} \frac{X F_{2}(X)}{(X-1)^{3} \sqrt{n-X}}+\frac{3 X^{2} f_{0}(X)}{(X-1)^{3} \sqrt{n-X}}-\frac{2 n X^{2}\{B-\psi(X)\}}{(X-1)^{2} \sqrt{n-X}}
\end{aligned}
$$

In the following, we set for simplicity

$$
\left\{\begin{aligned}
U_{0}(x) & :=\frac{x^{2} \sqrt{n-x}}{(1-x)^{5}} F_{2}(x)\{\lambda(x)-\tilde{\lambda}(X(x))\} \\
U_{1}(x) & :=\frac{3 x^{2} f_{0}(x)}{(x-1)^{3} \sqrt{n-x}}, \quad U_{2}(x):=\frac{2 n x^{2}\{B-\psi(x)\}}{(1-x)^{2} \sqrt{n-x}} \\
U_{3}(x) & :=\frac{n x F_{2}(x)}{(n-1)(x-1)^{3} \sqrt{n-x}},
\end{aligned}\right.
$$

and

$$
U_{4}(x):=U_{3}(X(x)), \quad U_{5}(x):=U_{1}(X(x)), \quad U_{6}(x):=U_{2}(X(x)),
$$

then we have

$$
V(x, X(x))=U_{0}(x)-U_{1}(x)+U_{2}(x)+U_{4}(x)+U_{5}(x)-U_{6}(x) .
$$

By means of Proposition 3, Lemma 2.1 in [12] and Lemma 7.1 we see easily that $U_{i}(x), \imath=0,1,2,4,5,6$, are all positive.

Now, taking an auxiliary parameter $t=n-X(x)$, we can put

$$
x=t^{n-1}\left(b_{1}+b_{2} t+\cdots\right) \text { near } x=0
$$

by the equality : $x(n-x)^{n-1}=X(n-X)^{n-1}=n t^{n-1}-t^{n}$. Therefore from the equality

$$
\left(b_{1}+b_{2} t+\cdots\right)\left(n-b_{1} t^{n-1}-b_{2} t^{n}+\cdots\right)^{n-1}=n-t,
$$

we obtain easily

$$
b_{1}=\frac{1}{n^{n-2}}, \quad b_{2}=-\frac{1}{n^{n-1}},
$$

hence

$$
x=t^{n-1}\left(\frac{1}{n^{n-2}}-\frac{t}{n^{n-1}}+\cdots\right),
$$

supposing $n>2$. Next, we shall compute $\lambda(x)-\tilde{\lambda}(X)$. From (1.4) and (7.1), we have 


$$
\begin{aligned}
\lambda(x)-\tilde{\lambda}(X) & =\log (n-x)+\frac{n-1}{n-x}-\log (n-X)-\frac{n X-1}{(n-1) X} \\
& =\log n+\frac{n-1}{n}+O\left(t^{n-1}\right)-\log t-\frac{n+1}{n}+O(t) \\
& =-\log t+\log n-\frac{2}{n}+O(t) .
\end{aligned}
$$

Hence we obtain

$$
\begin{aligned}
U_{0}(x) & =\left\{\sqrt{n} F_{2}(0)+O\left(t^{n-1}\right)\right\} x^{2}\left\{-\log t+\log n-\frac{2}{n}+O(t)\right\} \\
& =O\left(t^{2 n-2-m}\right), \quad 0<m<1,
\end{aligned}
$$

since we have

$$
\lim _{t \rightarrow+0} t^{m} \log t=0
$$

for any small positive constant $m$. Analogously we have

$$
U_{1}(x)=O\left(t^{2 n-2}\right), \quad U_{2}(x)=O\left(t^{2 n-2}\right) .
$$

Next, we shall compute $U_{4}(x), U_{5}(x), U_{6}(x)$. We have

$$
U_{4}(x)=\frac{n}{n-1} \frac{X F_{2}(X)}{(X-1)^{3} \sqrt{n-X}}=\frac{n}{(n-1) \sqrt{t}}=\left\{\frac{n F_{2}(n)}{(n-1)^{3}}-\left.\frac{d}{d X} \frac{X F_{2}(X)}{(X-1)^{3}}\right|_{x=n} t+\cdots\right\}
$$

and

$$
\left.\frac{d}{d X} \frac{X F_{2}(X)}{(X-1)^{3}}\right|_{x=n}=-\frac{(2 n+1) F_{2}(n)}{(n-1)^{4}}+\frac{n F_{2}{ }^{\prime}(n)}{(n-1)^{3}} .
$$

From (4.2) we have

$$
\begin{aligned}
& F_{2}(n)=-P_{2}(n) B=(n-1)^{2}(2 n-3) B, \\
& F_{2}{ }^{\prime}(n)=-P_{2}{ }^{\prime}(n) B=8(n-1) B
\end{aligned}
$$

and hence

$$
\left.\frac{d}{d X} \frac{X F_{2}(X)}{(X-1)^{3}}\right|_{x=n}=-\frac{\left(4 n^{2}-12 n-3\right) B}{(n-1)^{2}}
$$

Thus we obtain

$$
U_{4}(x)=\frac{n^{2}(2 n-3) B}{(n-1)^{2} \sqrt{t}}+\frac{n\left(4 n^{2}-12 n-3\right) B}{(n-1)^{3}} \sqrt{t}\{1+O(t)\}
$$

Next, we have

$$
U_{5}(x)=\frac{3 X^{2} f_{0}(X)}{(X-1)^{3} \sqrt{n-X}}=\frac{3}{\sqrt{t}}\left\{\frac{n^{2} f_{0}(n)}{(n-1)^{3}}-\left.\frac{d}{d X} \frac{X^{2} f_{0}(X)}{(X-1)^{3}}\right|_{x=n} t+\cdots\right\}
$$

and 


$$
\left.\frac{d}{d X} \frac{X^{2} f_{0}(X)}{(X-1)^{3}}\right|_{x=n}=-\frac{n(n+2) f_{0}(n)}{(n-1)^{4}}+\frac{n^{2} f_{0}^{\prime}(n)}{(n-1)^{3}} .
$$

From (2.10) we have $f_{0}(n)=(n-1) B, f_{0}{ }^{\prime}(n)=-B$ and hence

$$
\left.\frac{d}{d X} \frac{X^{2} f_{0}(X)}{(X-1)^{3}}\right|_{x=n}=-\frac{2 n(n+1) B}{(n-1)^{3}} \text {. }
$$

Thus we obtain

$$
U_{5}(x)=\frac{3 n^{2} B}{(n-1)^{2} \sqrt{t}}+\frac{6 n(n+1) B}{(n-1)^{3}} \sqrt{t}\{1+O(t)\} .
$$

Next, we have

$$
U_{6}(x)=\frac{2 n X^{2}\{B-\psi(X)\}}{(X-1)^{2} \sqrt{n-X}}=\frac{2 n}{\sqrt{t}}\left\{\frac{n^{2} B}{(n-1)^{2}}-\left.\frac{d}{d X} \frac{X^{2}\{B-\psi(X)\}}{(X-1)^{2}}\right|_{x=n} t+\cdots\right\}
$$

and

$$
\left.\frac{d}{d X} \frac{X^{2}\{B-\psi(X)\}}{(X-1)^{2}}\right|_{x=n}=-\frac{2 n B}{(n-1)^{3}} .
$$

Thus we obtain

$$
U_{6}(x)=\frac{2 n^{3} B}{(n-1)^{2} \sqrt{t}}+\frac{4 n^{2} B}{(n-1)^{3}} \sqrt{t}\{1+O(t)\} .
$$

By means of $(8.5) \sim(8.9)$, we have

$$
\begin{aligned}
V(x, X(x))= & O\left(t^{2 n-2-m}\right)+O\left(t^{2 n-2}\right)+\frac{B}{\sqrt{t}}\left\{\frac{n^{2}(2 n-3)}{(n-1)^{2}}+\frac{3 n^{2}}{(n-1)^{2}}-\frac{2 n^{3}}{(n-1)^{2}}\right\} \\
& +B \sqrt{t}\left\{\frac{n\left(4 n^{2}-12 n-3\right)}{(n-1)^{3}}+\frac{6 n(n+1)}{(n-1)^{3}}-\frac{4 n^{2}}{(n-1)^{3}}+O(t)\right\},
\end{aligned}
$$

i. e.

$$
V(x, X(x))=\frac{n\left(4 n^{2}-10 n+3\right) B}{(n-1)^{3}} \sqrt{t}(1+O(\iota)),
$$

which implies immediately

$$
\lim _{x \rightarrow+0} V(x, X(x))=0
$$

and $V(x, X(x))>0$ sufficiently near $x=0$, when $n>\frac{5+\sqrt{13}}{4}=2.15138 \cdots$.

Finally we shall prove the statement (iv). Taking an auxiliary parameter

$$
t=1-x,
$$

we can put near $x=1$ as

$$
X=1+b_{1} t+b_{2} t^{2}+\cdots .
$$

Substituting these into the equality 
we have

$$
(1-x)(n-x)^{n-2} d x=(1-X)(n-X)^{n-2} d X,
$$

$$
(n-1+t)^{n-2}=\left(b_{1}+b_{2} t+\cdots\right)\left(b_{1}+2 b_{2} t+\cdots\right)\left(n-1-b_{1} t-b_{2} t^{2}+\cdots\right)^{n-2},
$$

from which we obtain easily

$$
b_{1}=1, \quad b_{2}=\frac{2(n-2)}{3(n-1)}
$$

and hence

$$
X=1+t+\frac{2(n-2)}{3(n-1)} t^{2}+\cdots .
$$

We have

$$
\begin{aligned}
\frac{x^{2} \sqrt{n-x}}{(1-x)^{5}} & =\frac{\sqrt{n-1}}{t^{5}}\left(1-2 t+t^{2}\right)\left(1+\frac{t}{2(n-1)}-\frac{t^{2}}{8(n-1)^{2}}+\cdots\right) \\
& =\frac{\sqrt{n-1}}{t^{5}}\left\{1-\frac{4 n-5}{2(n-1)} t+\frac{8 n^{2}-24 n+15}{8(n-1)^{2}} t^{2}+\cdots\right\},
\end{aligned}
$$

and from (4.5) and (4.6)

$$
\begin{aligned}
F_{2}(x)= & -P_{2}(x) B+(n-x)^{n-1} P_{3}(x) \\
= & -P_{2}(1-t) B+B\left(1+\frac{t}{n-1}\right)^{n-1} P_{3}(1-t) \\
= & -B\left\{12(n-1)^{2}+2\left(2 n^{2}+3 n-5\right) t+(2 n+1) t^{2}\right\} \\
& +B\left\{1+t+\frac{n-2}{2(n-1)} t^{2}+\frac{(n-2)(n-3)}{6(n-1)^{2}} t^{3}+\frac{(n-2)(n-3)(n-4)}{24(n-1)^{3}} t^{4}+\cdots\right\} \\
& \times\left\{12(n-1)^{2}-2(n-1)(4 n-11) t+\left(2 n^{2}-10 n+11\right) t^{2}+(n-1) t^{3}\right\},
\end{aligned}
$$

i. e.

$$
F_{2}(1-t)=t^{4}\left\{\frac{n\left(n^{2}-n+1\right) B}{6(n-1)}+O(t)\right\} .
$$

Then, setting

$$
X=1+s, \quad s=t+\frac{2(n-2)}{3(n-1)} t^{2}+\cdots,
$$

we obtain from (1.4) and (7.1)

$$
\begin{aligned}
& \lambda(x)-\tilde{\lambda}(X)=\log (n-x)+\frac{n-1}{n-x}-\log (n-X)+\frac{1}{(n-1) X}-\frac{n}{n-1} \\
& =\log \left(1+\frac{t}{n-1}\right)+\frac{1}{1+\frac{t}{n-1}}-\log \left(1-\frac{s}{n-1}\right)+\frac{1}{(n-1)(1+s)}-\frac{n}{n-1}
\end{aligned}
$$




$$
\begin{aligned}
= & \left(\frac{t}{n-1}-\frac{t^{2}}{2(n-1)^{2}}+\cdots\right)+\left(1-\frac{t}{n-1}+-\frac{t^{2}}{(n-1)^{2}}-\cdots\right)-\frac{n}{n-1} \\
& +\left(\frac{s}{n-1}+\frac{s^{2}}{2(n-1)^{2}}+\cdots\right)+\left(\frac{1}{n-1}-\frac{s}{n-1}+\frac{s^{2}}{n-1}+\cdots\right) \\
= & \left(\frac{t^{2}}{2(n-1)^{2}}+\cdots\right)+\left(\frac{(2 n-1) s^{2}}{2(n-1)^{2}}+\cdots\right),
\end{aligned}
$$

hence

$$
\lambda(x)-\tilde{\lambda}(X)=t^{2}\left\{\frac{n}{(n-1)^{2}}+O(t)\right\} .
$$

Then, using (8.13), (8.14) and (8.15), we obtain

$$
\begin{aligned}
U_{0}(x) & =\frac{x^{2} \sqrt{n-x}}{(1-x)^{5}} F_{2}(x)\{\lambda(x)-\tilde{\lambda}(X(x))\} \\
& =t\left\{\frac{n^{2}\left(n^{2}-n+1\right)(n-1)^{n-7 / 2}}{6}+O(t)\right\} .
\end{aligned}
$$

Next, we have from (2.10)

$$
\begin{aligned}
f_{0}(x)= & (2 n-1-x) B-(n-x)^{n-1}\left\{n-x+(n-1) x^{2}\right\} \\
= & \{2(n-1)+t\} B-B\left(1+\frac{t}{n-1}\right)^{n-1}\left\{2(n-1)-(2 n-3) t+(n-1) t^{2}\right\} \\
= & B\{2(n-1)+t\}-B\left\{2(n-1)-(2 n-3) t+(n-1) t^{2}\right\} \\
& \times\left\{1+t+\frac{n-2}{2(n-1)} t^{2}+\frac{(n-2)(n-3)}{6(n-1)^{2}} t^{3}+\frac{(n-2)(n-3)(n-4)}{24(n-1)^{3}} t^{4}+\cdots\right\},
\end{aligned}
$$

i. e.

$$
f_{0}(1-t)=-\frac{n(2 n-1) B}{6(n-1)} t^{3}-\frac{n(n-2)(3 n-1) B}{12(n-1)^{2}} t^{4}+\cdots
$$

Hence, we obtain

$$
\begin{aligned}
U_{1}(x)= & \frac{3 x^{2} f_{0}(x)}{(x-1)^{3} \sqrt{n-x}}=-\frac{3\left(1-2 t+t^{2}\right)}{t^{3} \sqrt{n-1+t}} \cdot f_{0}(1-t) \\
= & \frac{1}{\sqrt{n-1}}\left(1-2 t+t^{2}\right)\left(1-\frac{t}{2(n-1)}+\cdots\right) \\
& \times\left(\frac{n(2 n-1) B}{2(n-1)}+\frac{n(n-2)(3 n-1) B}{4(n-1)^{2}} t+\cdots\right)
\end{aligned}
$$

i. e.

$$
U_{1}(x)=\frac{1}{\sqrt{n-1}}\left\{\frac{n(2 n-1) B}{2(n-1)}-\frac{n\left(5 n^{2}-3 n+1\right) B}{4(n-1)^{2}} t+\cdots\right\}
$$


Analogously, we obtain

$$
\begin{aligned}
U_{5}(x) & =U_{1}(X(x))=U_{1}(1+s) \\
& =\frac{1}{\sqrt{n-1}}\left\{\frac{n(2 n-1) B}{2(n-1)}+\frac{n\left(5 n^{2}-3 n+1\right) B}{4(n-1)^{2}} s+\cdots\right\},
\end{aligned}
$$

therefore

$$
U_{5}(x)=\frac{1}{\sqrt{n-1}}\left\{\frac{n(2 n-1) B}{2(n-1)}+\frac{n\left(5 n^{2}-3 n+1\right) B}{4(n-1)^{2}} t+\cdots\right\} .
$$

Next, we have

$$
\begin{aligned}
& \frac{x^{2}}{(1-x)^{2} \sqrt{n-x}}-\frac{X^{2}}{(X-1)^{2} \sqrt{n-X}}=\frac{1-2 t+t^{2}}{t^{2} \sqrt{n-1+t}}-\frac{1+2 s+s^{2}}{s^{2} \sqrt{n-1-s}} \\
= & \frac{1}{t^{2} \sqrt{n-1}}\left(1-2 t+t^{2}\right)\left\{1-\frac{t}{2(n-1)}+\frac{3 t^{2}}{8(n-1)^{2}}+\cdots\right\} \\
& -\frac{1}{s^{2} \sqrt{n-1}}\left(1+2 s+s^{2}\right)\left\{1+\frac{s}{2(n-1)}+\frac{3 s^{2}}{8(n-1)^{2}}+\cdots\right\} \\
= & \frac{1}{t^{2} \sqrt{n-1}}\left\{1-\frac{4 n-3}{2(n-1)} t+\frac{8 n^{2}-8 n+3}{8(n-1)^{2}} t^{2}+\cdots\right\} \\
& -\frac{1}{s^{2} \sqrt{n-1}}\left\{1+\frac{4 n-3}{2(n-1)} s+\frac{8 n^{2}-8 n+3}{8(n-1)^{2}} s^{2}+\cdots\right\},
\end{aligned}
$$

into which substituting (8.12) we obtain

$$
\begin{aligned}
& \frac{x^{2}}{(1-x)^{2} \sqrt{n-x}}-\frac{X^{2}}{(X-1)^{2} \sqrt{n-X}} \\
= & \frac{1}{t^{2} \sqrt{n-1}}\left\{1-\frac{4 n-3}{2(n-1)} t+\frac{8 n^{2}-8 n+3}{8(n-1)^{2}} t^{2}+\cdots\right\} \\
& -\frac{1}{t^{2} \sqrt{n-1}}\left\{1-\frac{4(n-2)}{3(n-1)} t+\cdots\right\}\left\{1+\frac{4 n-3}{2(n-1)} t+\cdots\right\} \\
= & \frac{1}{t}\left\{-\frac{8 n-1}{3(n-1)^{3 / 2}}+O(t)\right\} .
\end{aligned}
$$

Furthermore, by Lemma 4.1 in [11] we have

$$
B-\psi(x)=B-\psi(X)=t^{2}\left\{\frac{n(n-1)^{n-2}}{2}+O(t)\right\} \text { near } x=1 .
$$

Thus, we obtain

$$
U_{2}(x)-U_{6}(x)=t\left\{-\frac{n^{2}(8 n-1)(n-1)^{n-7 / 2}}{3}+O(t)\right\} .
$$

Next, using (8.14) we have 


$$
\begin{array}{r}
U_{4}(x)=\frac{n X F_{2}(X)}{(n-1)(X-1)^{3} \sqrt{n-X}}=\frac{n(1+s)}{(n-1) s^{3}} \cdot \frac{1}{\sqrt{n-1}}\left\{1+\frac{s}{2(n-1)}+\frac{3 s^{2}}{8(n-1)^{2}}+\cdots\right\} \\
\quad \times s^{4}\left\{\frac{n\left(n^{2}-n+1\right) B}{6(n-1)}+O(s)\right\}=s\left\{\frac{n^{2}\left(n^{2}-n+1\right)(n-1)^{n-7 / 2}}{6}+O(s)\right\}
\end{array}
$$

and hence

$$
U_{4}(x)=t\left\{\frac{n^{2}\left(n^{2}-n+1\right)(n-1)^{n-7 / 2}}{6}+O(t)\right\} .
$$

By means of (8.16), (8.18), (8.19), (8.20) and (8.21), we have near $x=1$

$$
\begin{aligned}
V(x, X(x))= & t\left\{\frac{n^{2}\left(n^{2}-n+1\right)(n-1)^{n-7 / 2}}{6}+O(t)\right\} \\
& -\frac{n(2 n-1)(n-1)^{n-5 / 2}}{2}+t\left\{\frac{n\left(5 n^{2}-3 n+1\right)(n-1)^{n-7 / 2}}{4}+O(t)\right\} \\
& +\frac{n(2 n-1)(n-1)^{n-5 / 2}}{2}+t\left\{\frac{n\left(5 n^{2}-3 n+1\right)(n-1)^{n-7 / 2}}{4}+O(t)\right\} \\
& +t\left\{-\frac{n^{2}(8 n-1)(n-1)^{n-7 / 2}}{3}+O(t)\right\} \\
& +t\left\{\frac{n^{2}\left(n^{2}-n+1\right)(n-1)^{n-7 / 2}}{6}+O(t)\right\} \\
= & t\left\{\frac{n\left(2 n^{3}-3 n^{2}-5 n+3\right)(n-1)^{n-7 / 2}}{6}+O(t)\right\}
\end{aligned}
$$

i. e.

$$
V(x, X(x))=\frac{n(2 n-1)\left(n^{2}-n-3\right)(n-1)^{n-\tau / 2}}{6} t\{1+O(t)\},
$$

where $x=1-t$, which implies immediately

$$
\lim _{x \rightarrow 1-0} V(x, X(x))=0
$$

and

$$
V(x, X(x))>0 \quad \text { sufficiently near } x=1,
$$

when $n>\frac{1+\sqrt{13}}{2}=2.30277 \cdots$.

Q.E. D.

\section{REFERENCES}

[1] S. S. Chern, M. do CARmo and S. Kobayashi, Minimal submanifolds of a sphere with second fundamental form of constant length, Functional Analysis and Related Fields, Springer-Verlag, 1970, 60-75.

[2] S. FURUYA, On periods of periodic solutions of a certain nonlinear differential equation, Japan-United States Seminar on Ordinary Differential and Functional 
Equations, Lecture Notes in Mathematics, Springer-Verlag, 243 (1971), 320-323.

[3] W. Y. Hsiang and H. B. Lawson, Jr., Minimal submanifolds of low cohomogeneity, J. Diff. Geometry, 5 (1970), 1-38.

[4] T. OTsuki, Minimal hypersurfaces in a Riemannian manifold of constant curvature, Amer. J. Math., 92 (1970), 145-173.

[5] T. Oтsuкi, On integral inequalities related with a certain nonlinear differential equation, Proc. Japan Acad., 48 (1972), 9-12.

[6] T. OTsuki, On a 2-dimensional Riemannian manifold, Differential Geometry, in honor of K. Yano, Kinokuniya, Tokyo, 1972, 401-414.

[7] T. Otsuki, On a family of Riemannian manifolds defined on an $m$-disk, Math. J. Okayama Univ., 16 (1973), 85-97.

[8] T. OTsuki, On a bound for periods of solutions of a certain nonlinear differential equation (I), J. Math. Soc. Japan, 26 (1974), 206-233.

[9] T. OTsukI, On a bound for periods of solutions of a certain nonlinear differential equation (II), Funkcialaj Ekvacioj, 17 (1974), 193-205.

[10] T. Otsuki, Geodesics of $O_{n}^{2}$ and an analysis on a related Riemann surface, Tôhoku Math. J., 28 (1976), 411-427.

[11] T. OTsuki, A certain property of geodesics of the family of Riemannian manifolds $O_{n}^{2}$ (I), Proc. of Japan-United States Seminar on Minımal Submanifolds, including Geodesics, Kaigai, Tokyo, 1978, 173-192.

[12] T. OTsuki, A certain property of geodesics of the family of Riemannian man1folds $O_{n}^{2}$ (II), Kodai Math. J., 2 (1979), 211-242.

[13] M. Maeda And T. Otsuki, Models of the Riemannian manifolds $O_{n}^{2}$ in the Lorentzian 4-space, J. Diff. Geometry, 9 (1974), 97-108.

[14] M. URABE, Computations of periods of a certain nonlinear autonomous oscilations, Study of algorithms of numerical computatıons, Sûrikaıseki Kenkyûsho Kôkyûroku, 149 (1972), 111-129 (Japanese).

Department of Mathematics SCIENCE University OF TOKYO WakamiYa-cho 26, Shinjuku-KU TOKYO, JAPAN 162 